\title{
Modelling the Steady State Current at the Inlaid Disc Microelectrode for Homogeneous Mediated Enzyme Catalysed Reactions
}

\author{
J. Galceran ${ }^{\mathrm{a}}$, S. L. Taylor ${ }^{\mathrm{b}}$ and P. N. Bartlett ${ }^{\mathrm{b} *}$
}

${ }^{a}$ Departament de Química, Universitat de Lleida, Rovira Roure, 177. E-25198 Lleida, SPAIN.

${ }^{b}$ Department of Chemistry, University of Southampton, Southampton, SO17 1BJ, UK.

*Corresponding author. Fax: +44 2380 593781. e-mail: p.n.bartlett@soton.ac.uk

\section{Abstract:}

The steady state currents at an inlaid microdisc electrode have been modelled for a redox mediated enzyme catalysed reaction (such as the glucose, glucose oxidase, ferrocene system) in which all the components are present in homogeneous solution and the reaction of the redox mediator at the electrode is assumed to be either reversible or mass transport limited. The numerical solution for the non-linear system in the axisymmetrical geometry of the inlaid disc is achieved by using the finite element method in an iterative scheme. The resulting concentration and reaction profiles provide useful insight into the process, and show -for some parameter values- the formation of an almost spherical, sharp reaction layer or shell, whose position can be approximately predicted. Conditions under which the scheme reverts to the pseudo-first order EC' mechanism and expressions to interpret the corresponding current are discussed. A simple approximate expression is worked out under the assumption of constant substrate concentration on the electrode surface. This approximate expression is useful in determining a combination of the parameters from the slope of measurements at low substrate concentration. The effect of some parameters is discussed in detail, leading to two suggestions which can improve the analytical performance: i) use of the lowest mediator concentration compatible with the background current and ii) use of the largest 
microelectrode which reaches steady state in a reasonable time. Experimental measurements using ferrocene monocarboxylic acid as the mediator for the glucose/glucose oxidase system agree with the model and confirm the order of magnitude of the previously reported parameter values.

Keywords: biosensors, simulation, microdisc electrode, homogeneous reaction, kinetic model, non-linearity, glucose sensor, Finite Element Method

\section{Introduction}

In order to obtain information on a new combination of mediator, enzyme and substrate, for a particular biosensor, the simple homogeneous system is commonly studied [13]. In such experiments all of the biosensor components can freely diffuse in solution and therefore a simple model can be applied in order to understand the system. A form of the homogeneous system is also employed by commercially available glucose biosensors [4], that use carbon paste electrodes containing mediator and enzyme that dissolves into a drop of blood (added to the electrode). A deeper understanding of the effects of the concurrent parameters on the measured current can therefore be helpful in improving biosensors.

The homogeneous enzyme system has been studied elsewhere for the one dimensional geometry corresponding to a macroelectrode [5-12]. Analytical expressions for rotating disc and cyclic voltammetry studies of the homogeneous system have been derived for the limiting cases $[7,11]$ corresponding to unsaturating and saturating substrate concentrations. Kinetic constants have been obtained by fitting experimental data to the analytical expressions in the appropriate case [13]. 
The study of currents at disc shaped electrodes is more difficult, not only because of their two-dimensional geometry, but also because of the singularity present at the inlaid discinsulator boundary which produces the so-called edge effect [14-19]. Some linear diffusionreaction systems have been studied at inlaid disc electrodes, such as the pseudo first-order EC' system, in either the transient [20,21] or steady state [22-26]. However, non-linearity (either of the full Michaelis-Menten type or of its simplified second-order form) is a key complication arising from the homogeneous enzymatic reaction.

The inclusion of homogeneous second-order kinetics at spherical electrodes has been reported [27-29]. Second-order kinetics at disc electrodes was successfully dealt with by Tutty $[17,30]$.

In this work we consider the Michaelis-Menten mechanism for the homogeneous enzymatic reaction which, through a mediator, produces a steady state current at the inlaid disc electrode. The organisation of the work is as follows. In section 1, the system is modelled with two partial differential equations involving four dimensionless parameters. In section 2, the outline of the numerical solution is described (simulation details are given in appendix A) together with an analysis of the profiles. In section 3, approximate expressions for the dimensionless current are suggested and discussed. In section 4, the influence of the bulk concentration, electrode radius and rate constants is shown. Section 5 is devoted to the comparison of the experimental data for glucose/glucose oxidase with simulated currents. Appendix B gathers a list of the main symbols used. 


\section{1.- Mathematical formulation of the enzyme model}

Let us consider a solution containing a substrate $\mathrm{S}$ which can be oxidised to a product $\mathrm{P}$ by the action of the oxidised form, $\mathrm{E}_{\mathrm{O}}$, of an enzyme:

$$
\mathrm{E}_{\mathrm{O}}+\mathrm{S} \underset{k_{-1}}{\stackrel{k_{1}}{\rightleftharpoons}}\left\{\mathrm{SE}_{\mathrm{O}}\right\} \stackrel{k_{\mathrm{cat}}}{\longrightarrow} \mathrm{E}_{\mathrm{R}}+\mathrm{P}
$$

Neglecting the diffusion of the complex in comparison to the kinetics terms, its steady state concentration can be computed as:

$$
\left[\left\{\mathrm{SE}_{0}\right\}\right]=\frac{k_{1}\left[\mathrm{E}_{0}\right][\mathrm{S}]}{k_{-1}+k_{c a t}}=\frac{\left[\mathrm{E}_{0}\right][\mathrm{S}]}{K_{\mathrm{M}}}
$$

where

$$
K_{\mathrm{M}} \equiv \frac{k_{-1}+k_{c a t}}{k_{1}}
$$

is the Michaelis-Menten constant. Dimensional concentrations (in mol $\mathrm{dm}^{-3}$ ) are denoted with the symbol of the species in between square brackets. Notice that [S] stands for the concentration of substrate, which in the glucose sensor is the concentration of the $\beta$-anomer of glucose and not the total concentration of glucose.

The mediator $A$ regenerates $E_{O}$ from its reduced form $E_{R}$ :

$$
m \mathrm{~A}+\mathrm{E}_{\mathrm{R}} \stackrel{k_{\mathrm{A}}}{\longrightarrow} m \mathrm{~B}+\mathrm{E}_{\mathrm{O}}
$$

(where $m$ is the stoichiometric number, usually 1 or 2 [8][17,27,30], of mediator molecules needed to regenerate the enzyme). Conversely, A is regenerated from its form $\mathrm{B}$ on the electrode surface (for the global scheme see Fig 1 ):

$\mathrm{B}-n_{\mathrm{e}} e^{-} \longrightarrow \mathrm{A}$ 
Let us label as the "bulk determined invariant" a well-known result [25,26,31-33]: if $f$ is a function satisfying the Laplace equation $\left(\nabla^{2} f=0\right)$ in a domain (e.g. the volume of the solution) with boundary conditions of no gradient of $f$ across any boundary (e.g. insulator) and a boundary condition corresponding to a "bulk" concentration value (i.e. a Dirichlet condition imposing $f=f^{*}$ at a finite or infinite distance from the electrode), then $f=f^{*}$ along all the domain. This property can be used in the mechanistic scheme considered here: i) for the total enzyme concentration, ii) for the substrate/product and iii) for the mediator, whatever the actual geometry of the system (electrode shape and size, insulator position, etc.).

i) Let $\left[E_{\Sigma}\right]$ be the total concentration of enzyme:

$$
\left[\mathrm{E}_{\Sigma}\right] \equiv\left[\mathrm{E}_{\mathrm{O}}\right]+\left[\mathrm{E}_{\mathrm{R}}\right]+\left[\left\{\mathrm{SE}_{\mathrm{O}}\right\}\right]
$$

If we assume that the diffusion coefficient of all of the enzymatic forms $\left(\mathrm{E}_{\mathrm{O}}, \mathrm{E}_{\mathrm{R}},\left\{\mathrm{SE}_{\mathrm{O}}\right\}\right)$ is the same, we can write its continuity equation as $\nabla^{2}\left[\mathrm{E}_{\Sigma}\right]=0$ (with the implicit assumption that diffusion is the only mass transport phenomenon), which together with boundary conditions of zero flux at any surface (there are neither sources nor sinks for the enzyme) and a fixed bulk concentration value at infinite distance from the electrode, allows the application of the "bulk determined invariant" and leads to the conclusion that $\left[E_{\Sigma}\right]$ is constant at any point in the solution. Moreover, due to the high molecular weight of the enzyme [34] (ca. 186000 Dalton), it is also reasonable to neglect diffusion of any enzymatic species [8] in front of the kinetic terms.

In the steady state, the continuity equation that models the system $[5,6,8,11]$ is

$$
D_{\mathrm{A}} \nabla^{2}[\mathrm{~A}]=\frac{m k_{\mathrm{A}} k_{\mathrm{cat}}\left[\mathrm{E}_{\Sigma}\right][\mathrm{S}][\mathrm{A}]}{k_{\mathrm{A}} K_{\mathrm{M}}[\mathrm{A}]+k_{\mathrm{A}}[\mathrm{S}][\mathrm{A}]+k_{\mathrm{cat}}[\mathrm{S}]}
$$


for the oxidized mediator A. $D_{\mathrm{A}}$ stands for the diffusion coefficient of A and $\nabla^{2}$ stands for the (dimensional) laplacian operator in the appropriate geometry. These equations correspond to the so-called "ping-pong" aerobic mechanism of glucose oxidase [35].

For the reduced mediator $\mathrm{B}$, the continuity equation is

$$
D_{\mathrm{B}} \nabla^{2}[\mathrm{~B}]=-\frac{m k_{\mathrm{A}} k_{\mathrm{cat}}\left[\mathrm{E}_{\Sigma}\right][\mathrm{S}][\mathrm{A}]}{k_{\mathrm{A}} K_{\mathrm{M}}[\mathrm{A}]+k_{\mathrm{A}}[\mathrm{S}][\mathrm{A}]+k_{\mathrm{cat}}[\mathrm{S}]}
$$

For the substrate S, by analogy with eqn. (7), we can write

$$
D_{\mathrm{S}} \nabla^{2}[\mathrm{~S}]=\frac{k_{\mathrm{A}} k_{\mathrm{cat}}\left[\mathrm{E}_{\Sigma}\right][\mathrm{S}][\mathrm{A}]}{k_{\mathrm{A}} K_{\mathrm{M}}[\mathrm{A}]+k_{\mathrm{A}}[\mathrm{S}][\mathrm{A}]+k_{\mathrm{cat}}[\mathrm{S}]}
$$

and for the product $\mathrm{P}$,

$$
D_{\mathrm{P}} \nabla^{2}[\mathrm{P}]=-\frac{k_{\mathrm{A}} k_{\mathrm{cat}}\left[\mathrm{E}_{\Sigma}\right][\mathrm{S}][\mathrm{A}]}{k_{\mathrm{A}} K_{\mathrm{M}}[\mathrm{A}]+k_{\mathrm{A}}[\mathrm{S}][\mathrm{A}]+k_{\text {cat }}[\mathrm{S}]}
$$

ii) Let us consider species $\mathrm{S}$ and $\mathrm{P}$. There is only interconversion between them without any sources or sinks, so that the "bulk determined invariant" applies. In effect, as the conversion takes place in the solution, their boundary conditions at any limiting surface are:

$D_{\mathrm{S}}\left(\frac{d[\mathrm{~S}]}{d n}\right)_{\text {boundary }}=-D_{\mathrm{P}}\left(\frac{d[\mathrm{P}]}{d n}\right)_{\text {boundary }}=0$

where $n$ is a coordinate normal to the boundary surface [32]. By adding (9) and (10), we obtain $\nabla^{2}\left(D_{\mathrm{S}}[\mathrm{S}]+D_{\mathrm{P}}[\mathrm{P}]\right)=0$, and taking into account the boundary conditions (11) and the fact that at a point remote from the electrode the bulk concentration of $\mathrm{P}$ is assumed to be 0 ,

$$
D_{\mathrm{S}}[\mathrm{S}]+D_{\mathrm{P}}[\mathrm{P}]=D_{\mathrm{S}}[\mathrm{S}]^{*}
$$

where the asterisk superscript indicates bulk conditions. 
iii) We can also apply the "bulk determined invariant" to a combination of the concentrations of the mediator species A and B. By adding (7) and (8), we obtain $\nabla^{2}\left(D_{\mathrm{A}}[\mathrm{A}]+D_{\mathrm{B}}[\mathrm{B}]\right)=0$, from which, together with the equality of the incoming and outcoming flux at any boundary

$$
D_{\mathrm{A}}\left(\frac{d[\mathrm{~A}]}{d n}\right)_{\text {boundary }}=-D_{\mathrm{B}}\left(\frac{d[\mathrm{~B}]}{d n}\right)_{\text {boundary }}
$$

one obtains:

$$
D_{\mathrm{A}}[\mathrm{A}]+D_{\mathrm{B}}[\mathrm{B}]=D_{\mathrm{B}}[\mathrm{B}]^{*}
$$

where the fact that $\mathrm{A}$ is not present at points remote from the electrode surface has been used.

Thus, by virtue of the "invariants" (12) and (14), which are just algebraic relationships, only two of the four differential eqns. (7)-(10) need to be solved.

If we further assume an electrochemically reversible reaction at the electrode surface [32]

$$
\frac{[\mathrm{A}]^{\mathrm{e}}}{[\mathrm{B}]^{\mathrm{e}}}=\exp \left[\frac{n_{\mathrm{e}} F}{R T}\left(E-E^{0}\right)\right] \equiv \delta
$$

where the superscript e stands for the electrode surface. Then, using eqn (14) at the electrode surface, one obtains,

$$
[\mathrm{A}]^{\mathrm{e}}=\frac{\delta D_{\mathrm{B}}[\mathrm{B}]^{*}}{\delta D_{\mathrm{A}}+D_{\mathrm{B}}}
$$

which is uniform on the electrode surface, as expected for an electroactive species [36]. Moreover, its value is independent of the actual geometry of the electrode and insulator [32] [33]. In the particular case when $\delta \rightarrow \infty$, we have diffusion limited conditions. Our results can then also be used for an irreversible redox couple under mass transport limited conditions. 
We define the dimensionless concentrations as

$$
\begin{aligned}
& A \equiv \frac{[\mathrm{A}]}{[\mathrm{A}]^{\mathrm{e}}}=\frac{\left(\delta D_{\mathrm{A}}+D_{\mathrm{B}}\right)}{\delta D_{\mathrm{B}}[\mathrm{B}]^{*}}[\mathrm{~A}] \\
& S \equiv \frac{[\mathrm{S}]}{[\mathrm{S}]^{*}}
\end{aligned}
$$

Having in mind the application to the disc electrode (where the electrode radius $a$ is the normalising distance), we introduce the following dimensionless parameters:

$\gamma_{\mathrm{B}} \equiv \frac{k_{\mathrm{A}} \delta D_{\mathrm{B}}[\mathrm{B}]^{*}}{k_{\text {cat }}\left(\delta D_{\mathrm{A}}+D_{\mathrm{B}}\right)}$

where the subscript $\mathrm{B}$ recalls that this parameter can be seen as a dimensionless bulk concentration of the mediator;

$$
\gamma_{\mathrm{S}} \equiv \frac{[\mathrm{S}]^{*}}{K_{\mathrm{M}}}
$$

which can be seen as a dimensionless bulk concentration of the substrate;

$$
\gamma_{\mathrm{E}} \equiv \frac{a^{2} k_{\mathrm{cat}}\left[\mathrm{E}_{\Sigma}\right]}{D_{\mathrm{S}} K_{\mathrm{M}}}
$$

which can be seen either as a dimensionless total concentration of the enzyme or as a dimensionless kinetic rate constant for the enzyme-substrate reaction; and

$$
\gamma_{\mathrm{r}} \equiv \frac{m D_{\mathrm{S}}[\mathrm{S}]^{*}\left(\delta D_{\mathrm{A}}+D_{\mathrm{B}}\right)}{D_{\mathrm{A}}[\mathrm{B}]^{*} \delta D_{\mathrm{B}}}
$$

which can be seen as a ratio of the effective diffusion contribution (or the diffusional availabilities) of species $\mathrm{S}$ and $\mathrm{B}$ to the homogeneous reaction. 
The continuity equations (7) and (9) in terms of the non-dimensional magnitudes just defined, now written explicitly for the axisymmetric geometry corresponding to the inlaid disc, become

$\frac{\partial^{2} A}{\partial r^{2}}+\frac{1}{r} \frac{\partial A}{\partial r}+\frac{\partial^{2} A}{\partial z^{2}}=\frac{\gamma_{\mathrm{E}} \gamma_{\mathrm{r}} \gamma_{\mathrm{B}} A S}{\gamma_{\mathrm{B}} A+\gamma_{\mathrm{B}} \gamma_{\mathrm{S}} A S+\gamma_{\mathrm{S}} S}$

and

$$
\frac{\partial^{2} S}{\partial r^{2}}+\frac{1}{r} \frac{\partial S}{\partial r}+\frac{\partial^{2} S}{\partial z^{2}}=\frac{\gamma_{\mathrm{E}} \gamma_{\mathrm{B}} A S}{\gamma_{\mathrm{B}} A+\gamma_{\mathrm{B}} \gamma_{\mathrm{S}} A S+\gamma_{\mathrm{S}} S}
$$

where $r$ and $z$ are dimensionless coordinates normalised with respect to the electrode radius $a$. Eqns. (23) and (24) constitute a non-linear system due to the presence of the "kinetic term" on the right hand side of both equations, which also acts as the coupling between them.

The boundary conditions can be written

$A=1 \quad z=0 \quad r \leq 1$

$\frac{\partial A}{\partial z}=0 \quad z=0 \quad r>1$

$\frac{\partial S}{\partial z}=0 \quad z=0 \quad \forall r$

$\frac{\partial A}{\partial r}=0 \quad \frac{\partial S}{\partial r}=0 \quad r=0 \quad \forall z$

$A=0 \quad S=1 \quad r=\infty \quad \forall z$

$A=0 \quad S=1 \quad z=\infty \quad \forall r$ 
As the response function (which could be labelled "flux" $\phi$ ), we use the current normalised with respect to the current obtained when there is the same amount of mediator but no enzyme:

$$
\phi \equiv \frac{I\left(\left[\mathrm{E}_{\Sigma}\right] \neq 0\right)}{I\left(\left[\mathrm{E}_{\Sigma}\right]=0\right)}=\frac{n_{\mathrm{e}} F a D_{\mathrm{A}} \int_{0}^{1}\left(\frac{\partial[\mathrm{A}]}{\partial z}\right)_{z=0} 2 \pi r \mathrm{~d} r}{4 n_{\mathrm{e}} F a D_{\mathrm{A}}\left(\frac{\delta D_{\mathrm{B}}}{\delta D_{\mathrm{A}}+D_{\mathrm{B}}}\right)[\mathrm{B}]^{*}}=-\frac{\pi}{2} \int_{0}^{1}\left(\frac{\partial A}{\partial z}\right)_{z=0} r \mathrm{~d} r
$$

where a re-scaling of the well-known expression for the current to a disk [26,37] has been used in the denominator.

It is worthwhile noticing that the flux (or normalised current) depends on just four independent dimensionless parameters (eqns. (19)-(22)) which are combinations of sixteen original physical parameters. It can be seen that $D_{\mathrm{P}}$ is not included in any of the set $\left(\gamma_{\mathrm{B}}, \gamma_{\mathrm{S}}, \gamma_{\mathrm{E}}\right.$ and $\gamma_{\mathrm{r}}$ ) and thus, as expected from the assumed irreversibility of the second half of the catalytic reaction (1), the current is independent of its value. There are alternative choices for the normalised parameters $[11,38]$; for instance, replacing $\gamma_{\mathrm{r}}$ with the combination $\gamma_{\mathrm{r}} \gamma_{\mathrm{B}} / \gamma_{\mathrm{S}}$ has the advantage of rendering three separate experimentally-controlled parameters $\left(\gamma_{\mathrm{B}}\right.$ through the mediator bulk concentration or the applied potential, $\chi_{s}$ through the bulk substrate concentration and $\gamma_{E}$ through the electrode radius or enzyme concentration) while the new parameter $\left(\gamma_{\mathrm{r}} \gamma_{\mathrm{B}} / \gamma_{\mathrm{S}}\right)$ would just be a relationship of physical constants (at constant temperature and pressure). However, we prefer to keep $\gamma_{r}$ because of the simplicity of the approximate expressions derived in section 3.2 


\section{2.- Numerical solution}

\subsection{Finite Element approach}

We describe here the outline of the method of solution, while details are given in appendix A. The problem of solving eqns. (23) and (24) with boundary conditions (25)-(30) can be re-formulated as a weighted residual minimisation, in an extension of our previous works [18,20,39-41]. We have taken linear interpolation functions $N_{i}$ and triangular elements [18] with an expanding mesh $[41,42]$. The non-linearity has been dealt with through an iterative scheme [10]: in one iteration a linearized substrate continuity equation is solved using concentration values of $S$ and $A$ for the non-linear factors in the kinetic term from the previous iteration. In the following iteration, the mediator continuity equation is solved using concentration values of $S$ and $A$ for the non-linear factors from the previous iteration. Iterations are repeated until desired convergence of the flux is fulfilled. A copy of the program is available from the authors upon request.

\subsection{Profiles}

The solid lines in Fig 2 stand for contour lines linking points where the nondimensional concentration $A$ has a common value. The shape of these lines is very similar to that for just one electroactive species diffusing towards a disc. For values close to the value reached at the electrode surface (see, for instance, isoline $A=0.9$ ) the iso-value line is practically parallel to the electrode surface at the centre of the electrode and then, for increasing $r$, bends progressively surrounding the electrode singularity $(r=1, z=0)$. For values close to the bulk value (see, for instance, isoline $A=0.05$ ) the iso-surface becomes practically spherical. 
Away from the electrode, the S-profile, depicted in Fig 2 with markers $\mathbf{o}$ and with italic labels, conforms to spherical diffusion (see, for instance, isoline $S=0.55$ ). Approaching the electrode, $S$-values decrease as $\mathrm{S}$ is consumed in its reaction with an increasing amount of A. It is noteworthy that, because $\mathrm{S}$ is not electroactive, the profiles end normal to the electrode surface (see, for instance, isoline $S=0.35)$. This level curve $(S=0.35)$ also indicates that depletion of $\mathrm{S}$ is at a maximum at the centre of the electrode.

Both profiles (for S and A) are essentially similar to those described by Tutty [30] for a second order reaction (i.e. with the kinetic terms in the right hand side of (23) and (24) having no denominator). As in the second order reaction case, $[\mathrm{S}]$ approaches the bulk value more slowly than $[\mathrm{A}]$.

\subsection{The reaction shell}

Fig 3 is a contour plot, for the same set of parameters used in Fig 2, of the values of the non-dimensional enzymatic kinetic term $\left(K_{\mathrm{E}}\right)$ given by the right hand side in eqn. (24):

$$
K_{\mathrm{E}} \equiv \frac{\gamma_{\mathrm{E}} \gamma_{\mathrm{B}} A S}{\gamma_{\mathrm{B}} A+\gamma_{\mathrm{B}} \gamma_{\mathrm{S}} A S+\gamma_{\mathrm{S}} S}
$$

This kind of plot [30] is indicative of the regions where the kinetic process between the reacting species is more relevant. It is seen in Fig 3 that $K_{\mathrm{E}}$ decreases away from the electrode in approximately spherical shells similar to the profiles of S and A seen in Fig. 2. The region with the maximum value of $K_{\mathrm{E}}$ lies close to $r \approx 1.25, z \approx 0$ and does not include the singularity $(r=1, z=0)$, where the steepest gradient of A is produced. The level curve $K_{\mathrm{E}}=$ 0.27 is not continuous, indicating that a less reactive region appears in front of the electrode centre, which can be ascribed to the low value of $S$ there (see Fig 2). 
Fig. 4 shows analogous $K_{\mathrm{E}}$ contours, now producing a rather sharp reaction region (in the shape of a shell) for the maximum reaction $K_{\mathrm{E}}$ values at an approximate radius $r_{\mathrm{R}} \approx 2.75$. This position is further away from the electrode than in Fig 3 due to a lower bulk concentration of substrate allowing a larger zone in which A diffuses with little reaction. The value of $K_{\mathrm{E}}$ in Fig. 4 falls steeply in the outer region and more smoothly in the inner region. An estimation of the radius of the reaction shell (or reaction layer) can be obtained noticing that, for infinitely fast reaction between $\mathrm{S}$ and $\mathrm{A}$, only one of the two species can be present at any point in space. Thus, the reaction would tend to be localized in a very thin shell (or reaction surface) where each incoming molecule of $\mathrm{S}$ from the outer region would react with $m$ molecules of A arriving from the inner region. Assuming a spherical shell with dimensional radius $a \cdot r_{\mathrm{R}}$, the diffusion limited steady state outer matter flux is:

$$
\text { flux of } \mathrm{S}_{\text {outer }}=2 \pi\left(r_{\mathrm{R}} a\right)^{2} D_{\mathrm{S}} \frac{[\mathrm{S}]^{*}}{r_{\mathrm{R}} a}
$$

while the inner flux can be estimated from the analogy between disc and sphere [43] applied to the steady state flux between two concentric spheres:

$$
\text { flux of } \mathrm{A}_{\text {inner }}=\frac{2}{\pi} 2 \pi a^{2} D_{\mathrm{A}} \frac{r_{\mathrm{R}} a[\mathrm{~A}]^{\mathrm{e}}}{\left(r_{\mathrm{R}} a-a\right) a}
$$

Imposing flux of $\mathrm{A}_{\text {inner }}=m\left(\right.$ flux of $\left.\mathrm{S}_{\text {outer }}\right)$, one obtains:

$$
r_{\mathrm{R}} \approx \frac{2}{\pi} \frac{D_{\mathrm{A}}[\mathrm{A}]^{\mathrm{e}}}{m D_{\mathrm{S}}[\mathrm{S}]^{*}}+1=\frac{2}{\pi \gamma_{\mathrm{r}}}+1
$$

which indicates that the limiting position of the sharp reaction shell depends only on the ratio parameter $\gamma_{\mathrm{r}}$. Estimation (35), applied with the parameters used to generate Fig. 4, yields $r_{\mathrm{R}} \approx$ 2.87, which is acceptably good. Application of (35) with the parameters used to generate Fig. 3 yields $r_{\mathrm{R}} \approx 1.62$ which is a worse estimation, as could be expected from the fact that the 
highly reactive zone is too close to the disc for the disc-sphere analogy to be sufficiently accurate.

\section{3.- Approximate expressions for the current}

\subsection{Uniform substrate concentration on the electrode}

Subtraction of $\gamma_{r}$ times eqn. (24) from eqn. (23) allows for the cancellation of their kinetic terms. Defining the following difference $\varepsilon(r, z) \equiv A(r, z)-\gamma_{\mathrm{r}} S(r, z)$ as a new formalspecies non-dimensional concentration, one can write the combination of eqn (24) and (23) as:

$\frac{\partial^{2} \varepsilon}{\partial r^{2}}+\frac{1}{r} \frac{\partial \varepsilon}{\partial r}+\frac{\partial^{2} \varepsilon}{\partial z^{2}}=0$

whose boundary conditions are combinations of those previously given (25)-(30) which are uncoupled to any other species concentration except for

$$
\mathcal{E}(r, z)=1-\gamma_{\mathrm{r}} S(r, 0) \quad z=0 \quad r \leq 1
$$

We have observed in the numerical simulations, that for a given set of parameters, the values of $S$ at the surface of the electrode vary over a relatively small range. For instance, with parameters corresponding to Figs 2 and $3, S(0,0)=0.338$ and $S(1,0)=0.379$; and with the parameters in Fig $4, S(0,0)=0.094$ and $S(1,0)=0.108$. Thus, we derive an approximation based in taking the substrate surface concentration as a constant:

$$
S(r, 0) \approx S^{\mathrm{e}} \quad r \leq 1
$$

By re-scaling the problem, taking

$$
\varepsilon_{1} \equiv \frac{\varepsilon+\gamma_{\mathrm{r}}}{1+\gamma_{\mathrm{r}}\left(1-S^{\mathrm{e}}\right)}
$$


the boundary conditions become those of semi-infinite diffusion towards an inlaid electrode, whose normalised solution flux is just unity. As

$$
\left(\frac{\partial \varepsilon}{\partial z}\right)_{z=0}=\left[1+\gamma_{\mathrm{r}}\left(1-S^{\mathrm{e}}\right)\right]\left(\frac{\partial \varepsilon_{1}}{\partial z}\right)_{z=0}
$$

we conclude:

$$
\phi \approx 1+\gamma_{\mathrm{r}}\left(1-S^{\mathrm{e}}\right)
$$

Fig 5 plots fluxes estimated with eqn. (41) using values of $S^{\mathrm{e}}$ obtained from numerical simulation ( $\square$ uses $S(0,0)$ as $S^{\mathrm{e}}, \times$ uses $S(1,0)$ and $\Delta$ uses the average $\left.(S(0,0)+S(0,1)) / 2\right)$. A good agreement with the simulated flux (shown as the solid line for three different radii) can be seen, especially when using the average concentration.

Let us consider the asymptotic behaviour of the flux when the bulk glucose concentration tends to 0 : then $S^{\mathrm{e}}$ tends to 0 , and eqn. (41) becomes

$$
\lim _{S^{\mathrm{e}} \rightarrow 0} \phi \approx 1+\gamma_{\mathrm{r}}
$$

This expression has also been represented in Fig 5 (discontinuous line). The physical reason why the flux in eqn. (42) only depends on the parameter $\gamma_{\mathrm{r}}$, is that, as $S^{\mathrm{e}}$ drops to 0 all the conversion of substrate into product is completed away from the electrode (in a more or less sharp reaction shell around $r_{\mathrm{R}}$ in (35)), because the kinetic rate is high enough (without relevance of its particular value), and, so, the flux only depends on a diffusion phenomenon which is expressed in $\gamma_{\mathrm{r}}$. 
However, for glucose concentrations which are not extremely low, some kind of estimate for $S^{\mathrm{e}}$ should be convenient for eqn. (41) to be useful. If we approximate the kinetic term in (23) by a linear dependence on $A$ :

$\frac{\partial^{2} A}{\partial r^{2}}+\frac{1}{r} \frac{\partial A}{\partial r}+\frac{\partial^{2} A}{\partial z^{2}}=\frac{\gamma_{\mathrm{E}} \gamma_{\mathrm{r}} \gamma_{\mathrm{B}} A S}{\gamma_{\mathrm{B}} A+\gamma_{\mathrm{B}} \gamma_{\mathrm{S}} A S+\gamma_{\mathrm{S}} S} \approx \kappa_{3} A$

we could use expressions available for steady state currents at the inlaid disc electrode under EC' conditions $[25,26]$. In order to assign an adequate value to $\kappa_{3}$, we recall that the S-profile in Fig 2 was smooth, so we can take $S \approx S^{\mathrm{e}}$. We postpone the selection of the $A$ value in the denominator of $\kappa_{3}$ and denote it by $\bar{A}$.

$$
\kappa_{3}=\frac{\gamma_{\mathrm{E}} \gamma_{\mathrm{r}} \gamma_{\mathrm{B}} S^{\mathrm{e}}}{\gamma_{\mathrm{B}} \bar{A}+\gamma_{\mathrm{B}} \gamma_{\mathrm{S}} \bar{A} S^{\mathrm{e}}+\gamma_{\mathrm{S}} S^{\mathrm{e}}}
$$

By equating the flux expressions given by (41) and eqn. (31) in ref. [26], we can numerically solve for $S$ in the algebraic equation

$$
1+\gamma_{\mathrm{r}}\left(1-S^{\mathrm{e}}\right)=\sqrt[3]{1+\frac{6}{\pi} \sqrt{\kappa_{3}}+\frac{3 \pi^{3}}{2^{6}} \kappa_{3}+\left(\frac{\pi}{4} \sqrt{\kappa_{3}}\right)^{3}}
$$

and then compute the flux with (41).

It is seen in Fig 6 that using this procedure with $\bar{A}=1 / 2$ (solid bullets $\bullet$ ) yields $5 \%$ accuracy for this particular case, while using $\bar{A}=1 / 3$ (marker + ) the least accurate is $3.3 \%$. The accuracy observed for other sets of parameters is similar. 


\subsection{The pseudo-first order EC' limit}

It is well known $[8,11,26]$ that the mechanism considered here -whatever the geometry- can revert to a pseudo-first order EC' scheme. In order to analyse which condition should be met, we start by noticing that the kinetic term in the mediator continuity eqn.

$$
D_{\mathrm{A}} \nabla^{2}[\mathrm{~A}]=m k_{\mathrm{A}}\left[\mathrm{E}_{\mathrm{R}}\right][\mathrm{A}]
$$

corresponding to reaction (4), becomes linear when $\left[E_{R}\right]$ can be taken as constant. In fact, $\left[E_{R}\right]$ decreases from its maximum value $\left[\mathrm{E}_{\Sigma}\right]$ in the bulk to its minimum value at the electrode surface, which can be evaluated as:

$$
\left[\mathrm{E}_{\mathrm{R}}\right]^{\mathrm{e}}=\frac{k_{\mathrm{cat}}\left[\mathrm{E}_{\Sigma}\right][\mathrm{S}]^{\mathrm{e}}}{k_{\mathrm{A}} K_{\mathrm{M}}[\mathrm{A}]^{\mathrm{e}}+k_{\mathrm{A}}[\mathrm{S}]^{\mathrm{e}}[\mathrm{A}]^{\mathrm{e}}+k_{\mathrm{cat}}[\mathrm{S}]^{\mathrm{e}}}
$$

If both values, $\left[E_{\Sigma}\right]$ and $\left[E_{R}\right]^{e}$, are to be close, one needs:

$$
k_{\mathrm{A}} K_{\mathrm{M}}[\mathrm{A}]^{\mathrm{e}}+k_{\mathrm{A}}[\mathrm{S}]^{\mathrm{e}}[\mathrm{A}]^{\mathrm{e}}<<k_{\text {cat }}[\mathrm{S}]^{\mathrm{e}}
$$

which can be re-written as

$$
[\mathrm{A}]^{\mathrm{e}}<<\frac{k_{\mathrm{cat}}[\mathrm{S}]^{\mathrm{e}}}{k_{\mathrm{A}} K_{\mathrm{M}}+k_{\mathrm{A}}[\mathrm{S}]^{\mathrm{e}}}=\frac{k_{\mathrm{cat}}}{k_{\mathrm{A}} K_{\mathrm{M}} /[\mathrm{S}]^{\mathrm{e}}+k_{\mathrm{A}}}
$$

The value of $[\mathrm{A}]^{\mathrm{e}}$ can be derived from experimentally controlled variables via eqn. (16) and for the usual case of limiting current is just $[\mathrm{B}]^{*}$. Unfortunately the precise value of $[\mathrm{S}]^{\mathrm{e}}$ is not known, but it is directly related with the substrate bulk concentration. Thus, expression (49) provides an indication of the concentration relationship to be met: high substrate (to ensure that $[\mathrm{S}]^{\mathrm{e}}$ is large) and low mediator concentration. If $[\mathrm{A}]^{\mathrm{e}}>\frac{k_{\text {cat }}}{k_{\mathrm{A}}}$, increasing $[\mathrm{S}]^{*}$ cannot eventually turn the scheme into pseudo-first order EC'.

The tendency towards the $1^{\text {st }}$ order EC' limit can be seen in Fig 7, where the horizontal dashed line stands for the current when solving the $1^{\text {st }}$ order EC' eqn.: 


$$
\frac{\partial^{2} A}{\partial r^{2}}+\frac{1}{r} \frac{\partial A}{\partial r}+\frac{\partial^{2} A}{\partial z^{2}}=\left(\frac{\gamma_{\mathrm{E}} \gamma_{\mathrm{r}} \gamma_{\mathrm{B}}}{\gamma_{\mathrm{S}}}\right) A=\left(\frac{m k_{\mathrm{A}}\left[\mathrm{E}_{\Sigma}\right] a^{2}}{D_{\mathrm{A}}}\right) A
$$

whose flux has been computed through the dual integral approach [26]. Notice that in the non-dimensional $1^{\text {st }}$ order kinetic constant, which is between brackets in eqn (50), neither $K_{\mathrm{M}}$ nor the substrate concentration are included.

The vertical dotted line in Fig 7 stands for an estimation of the right hand side of (49) taking $[\mathrm{S}]^{\mathrm{e}} \approx[\mathrm{S}]^{*}$. If the values of $k_{\mathrm{A}}, k_{\mathrm{cat}}, K_{\mathrm{M}}$ and $\gamma_{\mathrm{r}}$ are known, for a given experimental data couple (mediator concentration, flux) the validity of eqn. (49) can be tested by using (41):

$$
S^{\mathrm{e}} \approx 1-\frac{\phi-1}{\gamma_{\mathrm{r}}}
$$

\section{4.- Influence of the physical parameters on the normalised current}

\subsection{Effect of the bulk concentrations}

i) Substrate concentration: As seen in Figs. 5, 6 and 8, the flux is a continuously increasing function of the substrate concentration tending to a limiting plateau value. This can be physically explained in terms of the enhancement of the mediator cycling due to the substrate Michaelis-Menten mechanism, up to the saturating limit. At large enough substrate concentrations, for low mediator concentrations, the flux coincides with the pseudo-first order EC' value (see section 3.2).

The departure from $\phi=1$ at $[\mathrm{S}]^{*}$ close to 0 , is steep. The slope of the tangent to the plot of flux against substrate concentration in the limit of very low substrate concentration (see dashed line in Fig 6) can be derived from eqn. (42): 


$$
\lim _{S^{\mathrm{e}} \rightarrow 0} \phi \approx 1+\gamma_{r}=1+\left[\frac{m D_{\mathrm{S}}\left(\delta D_{\mathrm{A}}+D_{\mathrm{B}}\right)}{\mathrm{D}_{\mathrm{A}}[\mathrm{B}]^{*} \delta D_{\mathrm{B}}}\right][\mathrm{S}]^{*}
$$

The value of the slope could be used to determine the ratio $m D_{\mathrm{S}} / D_{\mathrm{A}}$ in a system where it is not known. Comparison of any three series with a common radius but different mediator concentrations in Fig 8, shows that the increase at $[\mathrm{S}]^{*}=0$ is more rapid, the lower the mediator concentration, which can be straightforwardly ascribed to the presence of $[\mathrm{B}]^{*}$ in the denominator of the slope corresponding to the factor in brackets in (52). On the other hand, from inspection of Fig 5, it is seen that the higher $\gamma_{\mathrm{E}}$ (which depends upon the electrode radius) the more the curve of flux against substrate concentration approaches the limiting straight line, as $\gamma_{\mathrm{E}}$ can be seen as a "dimensionless kinetic constant" whose higher value produces a greater depletion of the value of $S^{\mathrm{e}}$ from the bulk value.

ii) Mediator concentration: As shown in Fig 7 or in the comparison between parts a), b) and c) of Fig 8, the non-dimensional flux is, for a fixed substrate concentration, a continuously decreasing function of $[\mathrm{B}]^{*}$ between two asymptotic limits. This can be physically explained as follows: for a fixed amount of substrate, a decrease in $[\mathrm{B}]^{*}$ produces an increase of $[S]^{e}$, which increases the concentration $\left[E_{R}\right]$ (see eqns. (47) and (46)) and, thus, increases the efficiency of re-cycling of B. As a result the dimensionless current, which is normalised with respect to the diffusion limited current in the absence of enzyme catalysed reaction, increases as $[\mathrm{B}]^{*}$ decreases and approaches the $\mathrm{EC}^{\prime}$ limit which corresponds to $100 \%$ efficient recycling by the enzyme reaction and no loss of oxidised mediator, A, to the solution bulk. This suggests that, using the lowest mediator concentration compatible with the background current one could obtain a larger normalised response. Moreover, if condition (49) is met, expressions (either exact or approximate) [25,26] for pseudo-first order EC' could 
be used. As seen in Fig 7, the flux tends asymptotically to 1 when $[\mathrm{B}]^{*}$ increases sufficiently, as expected from the huge amount of mediator producing a current which then totally dominates the much smaller current arising from the enzymatic recycling mechanism.

\subsection{The effect of electrode radius}

The normalised current increases when $a$ increases, as seen in Figs 5 and 8. This can be understood because the radius $a$ only appears (as $a^{2}$ ) in the dimensionless parameter $\gamma_{E}$ (see eqn. (21)) where it multiplies $k_{\text {cat }}$ and $\left[\mathrm{E}_{\Sigma}\right]$, both of which are expected to increase the flux. In simple terms mass transport of oxidised mediator, A, away from the larger microelectrode is slower so more of the oxidised mediator reacts with the enzyme giving a greater dimensionless current. For those cases fulfilling condition (49), the linear dependence of the flux upon radius for small radii can easily be foreseen from its inclusion in the kinetic factor (the terms between brackets in eqn (50)) in the pseudo-first order EC' scheme corresponding to a very low kinetic factor

$$
\phi \approx 1+a \frac{2}{\pi} \sqrt{\frac{m k_{\mathrm{A}}\left[\mathrm{E}_{\Sigma}\right]}{D_{\mathrm{A}}}}
$$

or corresponding to large kinetic factor (which seems physically less realistic):

$$
\phi \approx \frac{\pi}{4}\left[a \sqrt{\frac{m k_{\mathrm{A}}\left[\mathrm{E}_{\Sigma}\right]}{D_{\mathrm{A}}}}+1\right]
$$

In both previous expressions, two terms have been retained in Phillips' asymptotic formulae [31]. Expression (53) could be used to determine the quantity inside the square root, by plotting, for sufficiently large amounts of substrate and sufficiently low concentrations of mediator (in order to revert to first order EC'), the flux against the radius (see Fig 9). If a small enough radius can be achieved with a reasonable accuracy, the corresponding first order 
kinetic constant is low, and eqn. (53) becomes adequate, so, the slope of the line as the radius tends to 0 allows the calculation of $m k_{\mathrm{A}}\left[\mathrm{E}_{\Sigma}\right] / D_{\mathrm{A}}[28]$.

From a practical point of view for the detection of an unknown concentration of glucose, the use of as large a microelectrode as possible, while keeping the time to reach steady state within a reasonable limit, will be beneficial.

\subsection{Effect of the rate}

As seen in Fig 10a, increasing $k_{\mathrm{A}}$ has its largest effect on the saturating steady state flux (plateau), where the substrate-enzyme reaction is no longer limiting, so that the rate and hence steady state flux is mainly determined by the mediator-enzyme kinetics.

In contrast, increasing $k_{\text {cat, }}$ see Fig $10 \mathrm{~b}$ ), also increases the steady state current but has a proportionally bigger effect on the current at low substrate concentrations where the enzyme substrate kinetics play a larger part in determining the current. For all substrate concentrations, increasing the turnover number of the enzyme increases the overall amount of reaction that can occur and so the steady state flux response increases.

The main impact of changing $K_{\mathrm{M}}$ is the modification of the curvature of the graph (see Fig 10c)), but the high and low substrate values for the different $K_{\mathrm{M}}$ values are still comparable. Lower $K_{\mathrm{M}}$ constants lead to a proportionally larger steady state flux at the lower unsaturated substrate concentrations (the plateau is reached at lower substrate concentrations). Changing $K_{\mathrm{M}}$, for this set of parameters, has less effect on the steady state flux near the 
saturating substrate region than either $k_{\mathrm{A}}$ or $k_{\mathrm{cat}}$. This is because on the plateau the kinetics are mainly governed by the rate of reaction of $A$ with $E_{R}$.

\section{5.- Experimental results}

A three electrode system was employed: platinum working microelectrode [44], SCE reference electrode and platinum gauze counter electrode. All experiments were carried out in $0.15 \mathrm{M}$ phosphate buffer $\mathrm{pH} 7$ (0.15 M disodium hydrogen orthophosphate and sodium dihydrogen orthophosphate, BDH, AnalaR grade). Ferrocene monocarboxylic acid (Avocado, $97 \%$, recrystallised from dichloromethane / petroleum ether at $40-60^{\circ} \mathrm{C}$ ) and glucose oxidase (E.C. 1.2.4.4., type VII, from Aspergillus niger supplied in a concentrated form by MediSense Inc.) were added to a cell and deoxygenated for $20 \mathrm{~min}$. Aliquots of ferrocene monocarboxylic acid and glucose (Aldrich) were added and the steady state current was recorded for microdisc electrodes of 10, 25 and $50 \mu \mathrm{m}$ diameter. In between each addition the solution was further deoxygenated for 5 min. Experimental results were collected for 4.37 $\mathrm{mM}$ and $1 \mathrm{mM}$ ferrocene monocarboxylic acid, for the various sized microdisc electrodes.

Glucose solutions were made from $\alpha$-D-glucose 24 hours before use, in order to allow the glucose isomers to mutarotate and reach the equilibrium ratio of $\beta$ to $\alpha$ anomers of 0.635 : 0.365. Taking into account that the $\beta$ anomer has a higher reaction rate with the enzyme than the $\alpha$ anomer by approximately two orders of magnitude and the slow interconversion rate $\left(6 \times 10^{-3} \mathrm{~s}^{-1}\right.$ in $\mathrm{pH} 7$ phosphate buffer/ TEATFB at $22^{\circ} \mathrm{C}$ [13]), the $\beta$-glucose concentration has been taken as the substrate concentration for the comparison between experimental and simulated data. 
Fig. 11 shows that the simulated curves lie through the experimental data points and therefore the model shows good correlation with the real system. The experimental currents decrease slightly in the high substrate region. This may be due to the increase in the viscosity of the solution with increasing glucose concentration. At high substrate the current should reach a plateau in this region, due to the substrate limited kinetics. Measuring the steady state current for a system with a lower mediator concentration gives a similar trend, as seen in Fig 12. In this instance, however, the fit is not quite as good, but shows that the kinetic parameters chosen for the model are in the correct range.

The scatter in the experimental data, particularly for the smaller electrode in figure 11 , is caused by the difficulty in measuring small increases in current over the diffusion current as substrate is added. The slight decrease in the current at high concentrations may be due to changes in the solution viscosity at high glucose concentration leading to a decrease in the diffusion coefficient of the mediator by approximately $10 \%$ over the full range of substrate concentration.

\section{Conclusions}

The non-linear equations corresponding to the model considered in this work (see Fig 1 and eqns. (7)-(10) ) can be solved with an iterative scheme of the Finite Element Method. Profiles for the various species and for the kinetic term show the similarities with spherical diffusion (spherical profiles far away from the electrode and formation of a "reaction shell" whose position can be estimated with eqn. (35)) as well as differences (distortions from spherical geometry close to the microelectrode). 
Approximating the concentration of substrate at the electrode surface by a constant leads to a good approximation of the flux, eqn (41). The flux can, then, be straightforwardly estimated for low substrate concentration, using eqn (42), which implies the linear relationship (52) between flux and substrate. For higher substrate concentrations, one can reach a reasonable estimate to the flux by making the following approximations in the kinetic term (right hand side in eqn (43)): approximating the substrate concentration by its concentration at the electrode surface and the mediator concentration by $\bar{A}=1 / 3$. This leads to the algebraic expression (45) which can be numerically solved, producing for the parameter values tested here, an accuracy of around 3\%.

In order to obtain a relatively high normalised signal for the detection of an unknown concentration of glucose, two recommendations arise from this work: i) use the smallest concentration of mediator sufficient to allow accurate measurement of the current above the background current and ii) use the largest microelectrode radius compatible with the practical attainment of steady state conditions.

Eqn. (49) provides a condition for pseudo-first order EC' to apply, which implies high substrate and low mediator. When condition (49) is met, the linear relationship (53) between the flux and sufficiently small electrode radii will hold.

The comparison of simulated steady state flux for the inlaid microdisc model with experimental measurements demonstrates that the model behaves in the correct manner and calculates flux values in the correct region. Secondly, using previously derived simulation parameters [13] the model shows good correlation with the experimental values, and this 
reinforces the accuracy of these parameters. It also shows that the experimental errors exceed those of the simulation by quite a large margin.

Finally, this study has shown that the combination of finite element simulation and analytical analysis leads to a deeper appreciation of the coupled non-linear diffusion-reaction system and the factors which determine its behaviour.

\section{Acknowledgements}

J.G. gratefully acknowledges support of this research by the Spanish Ministry of Education and Culture (DGICYT: Project PB96-0379 and Convenio S.E.U.I.D.-Royal Society), by the "Generalitat de Catalunya" (1999BEAI400019; 2000BEAI400053), by the "Universitat de Lleida"(1997) and by the "Ajuntament de Lleida". We thank EPSRC for financial support (grant GR/K 14704) and MediSense Inc. for a studentship for S.L.T.

\section{Appendix A: Finite Element formulation of the problem.}

Let us begin with the substrate equation (24). If $\omega$ stands for the weighting function,

$0=\int_{V} \omega\left(\frac{\gamma_{\mathrm{E}} \gamma_{\mathrm{B}} A S}{\gamma_{\mathrm{B}} A+\gamma_{\mathrm{B}} \gamma_{\mathrm{S}} A S+\gamma_{\mathrm{S}} S}-\frac{\partial^{2} S}{\partial r^{2}}-\frac{1}{r} \frac{\partial S}{\partial r}-\frac{\partial^{2} S}{\partial z^{2}}\right) \mathrm{d} V$

where $V$ represents the domain volume.

Integration by parts and application of the boundary conditions leads to

$0=\int_{V}\left(\frac{\partial \omega}{\partial r} \frac{\partial S}{\partial r}+\frac{\partial \omega}{\partial z} \frac{\partial S}{\partial z}+\frac{\omega \gamma_{\mathrm{E}} \gamma_{\mathrm{B}} A S}{\gamma_{\mathrm{B}} A+\gamma_{\mathrm{B}} \gamma_{\mathrm{S}} A S+\gamma_{\mathrm{S}} S}\right) \mathrm{d} V$ 
An analogous equation can be written for $A$ :

$$
0=\int_{V}\left(\frac{\partial \omega}{\partial r} \frac{\partial A}{\partial r}+\frac{\partial \omega}{\partial z} \frac{\partial A}{\partial z}+\frac{\omega \gamma_{\mathrm{E}} \gamma_{\mathrm{r}} \gamma_{\mathrm{B}} A S}{\gamma_{\mathrm{B}} A+\gamma_{\mathrm{B}} \gamma_{\mathrm{S}} A S+\gamma_{\mathrm{S}} S}\right) \mathrm{d} V
$$

Discretising the unknown functions as a sum of interpolation functions $N_{\mathrm{j}}$ that multiply the function values $S_{j}$ and $A_{j}$ at $\ell$ chosen nodes (common to both species),

$$
S=\sum_{j=1}^{\ell} S_{j} N_{j} \quad A=\sum_{j=1}^{\ell} A_{j} N_{j}
$$

and taking the interpolation functions $N_{i}$ as the weighting functions $\omega$, eqn. (A-2) becomes, for each unknown node i,

$$
0=\sum_{j=1}^{\ell} S_{j} \int_{V}\left(\frac{\partial N_{i}}{\partial r} \frac{\partial N_{j}}{\partial r}+\frac{\partial N_{i}}{\partial z} \frac{\partial N_{j}}{\partial z}\right) \mathrm{d} V+\sum_{j=1}^{\ell} S_{j} \int_{V}\left(\frac{\gamma_{\mathrm{E}} \gamma_{\mathrm{B}} A}{\gamma_{\mathrm{B}} A+\gamma_{\mathrm{B}} \gamma_{\mathrm{S}} A S+\gamma_{\mathrm{S}} S} N_{i} N_{j}\right) \mathrm{d} V
$$

where the discretisation has not been applied to the non-linear term inside the last integral. Due to this non-linearity, we have not solved the steady-state problem in one step, but through iteration of two linear systems [10]. In order to obtain an iterative scheme where a linear solution is found each time, we approximate this non-linear "kinetic factor" as a constant within each integration element, using the nodal values from the previous iteration

$$
\frac{\gamma_{\mathrm{E}} \gamma_{\mathrm{B}} A}{\gamma_{\mathrm{B}} A+\gamma_{\mathrm{B}} \gamma_{\mathrm{S}} A S+\gamma_{\mathrm{S}} S} \approx \frac{1}{2}\left(\frac{\gamma_{\mathrm{E}} \gamma_{\mathrm{B}} A_{i}}{\gamma_{\mathrm{B}} A_{i}+\gamma_{\mathrm{B}} \gamma_{\mathrm{S}} A_{i} S_{i}+\gamma_{\mathrm{S}} S_{i}}+\frac{\gamma_{\mathrm{E}} \gamma_{\mathrm{B}} A_{j}}{\gamma_{\mathrm{B}} A_{j}+\gamma_{\mathrm{B}} \gamma_{\mathrm{S}} A_{j} S_{j}+\gamma_{\mathrm{S}} S_{j}}\right)
$$

This choice has the advantage of keeping the symmetry of the matrices.

Let $n_{\mathrm{A}}$ and $n_{\mathrm{S}}$ represent the number of unknown nodes of $\mathrm{A}$ and $\mathrm{S}$ respectively and let us ascribe to the indices of the nodes corresponding to Dirichlet boundary conditions (either at the bulk or at the electrode surface) values greater than $n_{\mathrm{A}}$ and $n_{\mathrm{S}}$, respectively. Gathering unknown nodes on the left and known nodes on the right hand side, eqn.(A-5) becomes 


$$
\begin{aligned}
& \sum_{j=1}^{n_{\mathrm{S}}} S_{j} \int_{V}\left(\frac{\partial N_{i}}{\partial r} \frac{\partial N_{j}}{\partial r}+\frac{\partial N_{i}}{\partial z} \frac{\partial N_{j}}{\partial z}\right) \mathrm{d} V+\sum_{j=1}^{n_{\mathrm{S}}} \frac{S_{j}}{2}\left(\frac{\gamma_{\mathrm{E}} \gamma_{\mathrm{B}} A_{i}}{\gamma_{\mathrm{B}} A_{i}+\gamma_{\mathrm{B}} \gamma_{\mathrm{S}} A_{i} S_{i}+\gamma_{\mathrm{S}} S_{i}}+\frac{\gamma_{\mathrm{E}} \gamma_{\mathrm{B}} A_{\mathrm{j}}}{\gamma_{\mathrm{B}} A_{j}+\gamma_{\mathrm{B}} \gamma_{\mathrm{S}} A_{j} S_{j}+\gamma_{\mathrm{S}} S_{j}}\right) \int_{V} N_{i} N_{j} \mathrm{~d} V \\
& =-\sum_{j=n_{\mathrm{S}}+1}^{\ell} \int\left(\frac{\partial N_{i}}{\partial r} \frac{\partial N_{j}}{\partial r}+\frac{\partial N_{i}}{\partial z} \frac{\partial N_{j}}{\partial z}\right) \mathrm{d} V-\sum_{j=n_{\mathrm{S}}+1}^{\ell} \frac{1}{2}\left(\frac{\gamma_{\mathrm{E}} \gamma_{\mathrm{B}} A_{i}}{\gamma_{\mathrm{B}} A_{i}+\gamma_{\mathrm{B}} \gamma_{\mathrm{S}} A_{i} S_{i}+\gamma_{\mathrm{S}} S_{i}}\right) \int_{V} N_{i} N_{j} \mathrm{~d} V
\end{aligned}
$$

where the boundary conditions (29) and (30) have been applied. In each iteration step eqn. (A-7) is solved as a linear system for $S_{j}$.

Analogously, for the mediator

$$
\begin{aligned}
& \sum_{j=1}^{n_{\mathrm{A}}} A_{j} \int\left(\frac{\partial N_{i}}{\partial r} \frac{\partial N_{j}}{\partial r}+\frac{\partial N_{i}}{\partial z} \frac{\partial N_{j}}{\partial z}\right) \mathrm{d} V+\sum_{j=1}^{n_{\mathrm{A}}} \frac{A_{j}}{2}\left(\frac{\gamma_{\mathrm{E}} \gamma_{r} \gamma_{\mathrm{B}} S_{i}}{\gamma_{\mathrm{B}} A_{i}+\gamma_{\mathrm{B}} \gamma_{\mathrm{S}} A_{i} S_{i}+\gamma_{\mathrm{S}} S_{i}}+\frac{\gamma_{\mathrm{E}} \gamma_{r} \gamma_{\mathrm{B}} S_{j}}{\gamma_{\mathrm{B}} A_{j}+\gamma_{\mathrm{B}} \gamma_{\mathrm{S}} A_{j} S_{j}+\gamma_{\mathrm{S}} S_{j}}\right) \int_{V} N_{i} N_{j} \mathrm{~d} V \\
& =-\sum_{j=n_{\mathrm{A}}+1}^{\ell} A_{j} \int\left(\frac{\partial N_{i}}{\partial r} \frac{\partial N_{j}}{\partial r}+\frac{\partial N_{i}}{\partial z} \frac{\partial N_{j}}{\partial z}\right) \mathrm{d} V-\sum_{j=n_{\mathrm{A}}+1}^{\ell} \frac{A_{j}}{2}\left(\frac{\gamma_{\mathrm{E}} \gamma_{r} \gamma_{\mathrm{B}} S_{i}}{\gamma_{\mathrm{B}} A_{i}+\gamma_{\mathrm{B}} \gamma_{\mathrm{S}} A_{i} S_{i}+\gamma_{\mathrm{S}} S_{i}}+\frac{\gamma_{\mathrm{E}} \gamma_{r} \gamma_{\mathrm{B}} S_{j}}{\gamma_{\mathrm{B}} A_{j}+\gamma_{\mathrm{B}} \gamma_{\mathrm{S}} A_{j} S_{j}+\gamma_{\mathrm{S}} S_{j}}\right) \int_{V} N_{i} N_{j} \mathrm{~d} V
\end{aligned}
$$

\section{Iterative process}

When the values used as first guesses in the kinetic factor are far from the true steadystate solution, matrices can loose their positive definiteness. In order to avoid this effect, we have introduced an application fraction $q_{\text {appl }}$ (or damping of the kinetics) which multiplies the non-linear terms in (A-7) and (A-8):

$$
\begin{aligned}
& \sum_{j=1}^{n_{\mathrm{S}}} S_{j} \int\left(\frac{\partial N_{i}}{\partial r} \frac{\partial N_{j}}{\partial r}+\frac{\partial N_{i}}{\partial z} \frac{\partial N_{j}}{\partial z}\right) \mathrm{d} V+q_{\text {appl }} \sum_{j=1}^{n_{\mathrm{S}}} \frac{S_{j}}{2}\left(\frac{\gamma_{\mathrm{E}} \gamma_{\mathrm{B}} A_{i}}{\gamma_{\mathrm{B}} A_{i}+\gamma_{\mathrm{B}} \gamma_{\mathrm{S}} A_{i} S_{i}+\gamma_{\mathrm{S}} S_{i}}+\frac{\gamma_{\mathrm{E}} \gamma_{\mathrm{B}} A_{j}}{\gamma_{\mathrm{B}} A_{j}+\gamma_{\mathrm{B}} \gamma_{\mathrm{S}} A_{j} S_{j}+\gamma_{\mathrm{S}} S_{j}}\right) \int_{V} N_{i} N_{j} \mathrm{~d} V \\
& =-\sum_{j=n_{\mathrm{S}}+1}^{\ell} \int\left(\frac{\partial N_{i}}{\partial r} \frac{\partial N_{j}}{\partial r}+\frac{\partial N_{i}}{\partial z} \frac{\partial N_{j}}{\partial z}\right) \mathrm{d} V-q_{\text {appl }} \sum_{j=n_{\mathrm{S}}+1}^{\ell} \frac{1}{2}\left(\frac{\gamma_{\mathrm{E}} \gamma_{\mathrm{B}} A_{i}}{\gamma_{\mathrm{B}} A_{i}+\gamma_{\mathrm{B}} \gamma_{\mathrm{S}} A_{i} S_{i}+\gamma_{\mathrm{S}} S_{i}}\right) \int_{V} N_{i} N_{j} \mathrm{~d} V
\end{aligned}
$$

$$
\begin{aligned}
& \sum_{j=1}^{n_{\mathrm{A}}} A_{j} \int\left(\frac{\partial N_{i}}{\partial r} \frac{\partial N_{j}}{\partial r}+\frac{\partial N_{i}}{\partial z} \frac{\partial N_{j}}{\partial z}\right) \mathrm{d} V+q_{\text {appl }} \sum_{j=1}^{n_{\mathrm{A}}} \frac{A_{j}}{2}\left(\frac{\gamma_{\mathrm{E}} \gamma_{r} \gamma_{\mathrm{B}} S_{i}}{\gamma_{\mathrm{B}} A_{i}+\gamma_{\mathrm{B}} \gamma_{\mathrm{S}} A_{i} S_{i}+\gamma_{\mathrm{S}} S_{i}}+\frac{\gamma_{\mathrm{E}} \gamma_{r} \gamma_{\mathrm{B}} S_{j}}{\gamma_{\mathrm{B}} A_{j}+\gamma_{\mathrm{B}} \gamma_{\mathrm{S}} A_{j} S_{j}+\gamma_{\mathrm{S}} S_{j}}\right) \int_{V} N_{i} N_{j} \mathrm{~d} V \\
& =-\sum_{j=n_{\mathrm{A}}+1}^{\ell} A_{j} \int\left(\frac{\partial N_{i}}{\partial r} \frac{\partial N_{j}}{\partial r}+\frac{\partial N_{i}}{\partial z} \frac{\partial N_{j}}{\partial z}\right) \mathrm{d} V-q_{\text {appl }} \sum_{j=n_{\mathrm{A}}+1}^{\ell} \frac{A_{j}}{2}\left(\frac{\gamma_{\mathrm{E}} \gamma_{r} \gamma_{\mathrm{B}} S_{i}}{\gamma_{\mathrm{B}} A_{i}+\gamma_{\mathrm{B}} \gamma_{\mathrm{S}} A_{i} S_{i}+\gamma_{\mathrm{S}} S_{i}}+\frac{\gamma_{\mathrm{E}} \gamma_{r} \gamma_{\mathrm{B}} S_{j}}{\gamma_{\mathrm{B}} A_{j}+\gamma_{\mathrm{B}} \gamma_{\mathrm{S}} A_{j} S_{j}+\gamma_{\mathrm{S}} S_{j}}\right) \int_{V} N_{i} N_{j} \mathrm{~d} V
\end{aligned}
$$

$q_{\text {appl }}$ typically doubles during the 6 first iterations and reaches unity from the sixth iteration on. In each iteration eqn. (A-9) is solved in $S_{j}$ taking as values for $A_{j}$ and $S_{j}$ in the kinetic 
terms those found in the previous iteration. Then eqn. (A-10) (with the same $q_{\text {appl }}$ ) is solved for $A_{\mathrm{j}}$ using the $S_{\mathrm{j}}$ values and the $A_{\mathrm{j}}$ values from the previous iteration in the kinetic term. Typically, fifteen iterations (for six of which $q_{\mathrm{appl}}<1$ ) are enough to obtain a difference in the dimensionless currents between two iterations of less than 1 in $10^{5}$.

\section{Appendix B: List of Symbols}

\begin{tabular}{|l|l|l|}
\hline Symbol & Meaning & Equation(s) involved \\
\hline$*$ & bulk superscript & $(12),(14)$ \\
\hline$\nabla^{2}$ & dimensional laplacian operator & $(7),(8),(9),(10)$ \\
\hline$[\mathrm{X}]$ & $\begin{array}{l}\text { dimensional concentration of species X: A, } \\
\text { B, S, P, } \mathrm{E}_{\mathrm{R}}, \text { etc. }\end{array}$ & $(12),(14),(46)$ \\
\hline$a$ & dimensional electrode radius & $(21),(53)$ \\
\hline $\mathrm{A}$ & oxidised form of mediator species & $(4),(5)$ \\
\hline$A$ & dimensionless concentration of mediator A & $(17),(23)$ \\
\hline$A_{j}$ & dimensionless concentration of A at node j & $(\mathrm{A}-4)$ \\
\hline $\bar{A}$ & estimated average $A$-value & $(44)$ \\
\hline $\mathrm{B}$ & reduced form of mediator & $(4),(5)$ \\
\hline$D_{\mathrm{X}}$ & $\begin{array}{l}\text { diffusion coefficient of species X: } \\
\text { A, B, S, P, etc. }\end{array}$ & $(7),(8),(9),(10)$ \\
\hline $\mathrm{e}$ & electrode surface superscript & $(15),(38),(47)$ \\
\hline $\mathrm{E}_{\mathrm{O}}$ & oxidised enzyme & $(1),(4)$ \\
\hline $\mathrm{E}_{\mathrm{R}}$ & reduced enzyme & $(1),(4)$ \\
\hline$\left[\mathrm{E}_{\Sigma}\right]$ & total enzyme concentration & $(6),(21)$ \\
\hline$E, E^{\mathrm{o}}$ & electrode potentials & $(15)$ \\
\hline$f$ & $\begin{array}{l}\text { generic function fulfilling “bulk determined } \\
\text { invariant” }\end{array}$ & see below eqn. (5) \\
\hline$F$ & Faraday constant & $(15)$ \\
\hline$I$ & dimensional current & $(31)$ \\
\hline$k_{1}, k_{-1}$ & forward and backward rate constants & $(1),(2)$ \\
\hline$k_{\mathrm{A}}$ & mediator-enzyme rate constant & $(4),(7),(8),(19)$ \\
\hline$k_{\mathrm{cat}}$ & catalytic constant & $(1),(2),(7),(8),(19),(21)$ \\
\hline$K_{\mathrm{E}}$ & kinetic term & $(32)$ \\
\hline$K_{\mathrm{M}}$ & Michaelis-Menten constant & $(2),(3),(7),(8),(20),(21)$ \\
\hline$\ell$ & total number of nodes & $(4),(22)$ \\
\hline$m$ & stoichiometric number & $(5)$ \\
\hline$n_{\mathrm{e}}$ & $\begin{array}{l}\text { number of electrons exchanged at the } \\
\text { electrode surface }\end{array}$ & $(\mathrm{A}-7)$ \\
\hline$n_{\mathrm{A}}$ & number of nodes where $A$ is unknown & $(\mathrm{A}-7)$ \\
\hline$n_{\mathrm{S}}$ & number of nodes where $S$ is unknown \\
\hline$N_{j}$ & interpolation function corresponding to node & $\mathrm{A}-4)$ \\
\hline & & \\
\hline
\end{tabular}




\begin{tabular}{|l|l|l|}
\hline & $j$ & \\
\hline $\mathrm{P}$ & product & $(1)$ \\
\hline$q_{\text {appl }}$ & $\begin{array}{l}\text { factor for progressive application of the } \\
\text { kinetic term (or damping) }\end{array}$ & $(\mathrm{A}-9),(\mathrm{A}-10)$ \\
\hline$r$ & dimensionless radial co-ordinate & $(23),(24)$ \\
\hline$r_{\mathrm{R}}$ & dimensionless reaction shell radius & $(35)$ \\
\hline$R$ & gas constant & $(15)$ \\
\hline $\mathrm{S}$ & substrate & $(1)$ \\
\hline$S$ & dimensionless substrate concentration & $(18),(24)$ \\
\hline$S_{j}$ & dimensionless concentration of S at node $\mathrm{j}$ & $(\mathrm{A}-4)$ \\
\hline$\left\{\mathrm{SE}_{\mathrm{O}}\right\}$ & substrate-enzyme complex & $(1),(2)$ \\
\hline$T$ & temperature & $(15)$ \\
\hline$V$ & volume & $(\mathrm{A}-1)$ \\
\hline$z$ & dimensionless axial co-ordinate & $(23),(24)$ \\
\hline$\gamma_{\mathrm{B}}$ & $\begin{array}{l}\text { dimensionless bulk mediator concentration } \\
\text { parameter }\end{array}$ & $(19)$ \\
\hline$\gamma_{\mathrm{E}}$ & $\begin{array}{l}\text { dimensionless total enzyme concentration } \\
\text { parameter (or dimensionless kinetic rate } \\
\text { constant) }\end{array}$ & $(21)$ \\
\hline$\gamma_{\mathrm{r}}$ & $\begin{array}{l}\text { ratio of substrate and mediator diffusion } \\
\text { availability }\end{array}$ & $(22)$ \\
\hline$\gamma_{\mathrm{S}}$ & $\begin{array}{l}\text { dimensionless bulk substrate concentration } \\
\text { parameter }\end{array}$ & $(20)$ \\
\hline$\delta$ & dimensionless electrode potential & $(15),(19)$ \\
\hline$\varepsilon \equiv A-\gamma_{\mathrm{r}} S, \varepsilon_{1}$ & formal species & $(36),(37)$ \\
\hline$\kappa_{3}$ & equivalent first order EC' constant & $(43)$ \\
\hline$\phi$ & dimensionless current or flux & $(31)$ \\
\hline$\omega$ & weighting function in FEM & $(\mathrm{A}-1)$ \\
\hline
\end{tabular}




\section{Reference List}

[1] A.E.G.Cass, G.Davis, G.D.Francis, H.A.O.Hill, W.J.Aston, I.J.Higgins, E.V.Plotkin, L.D.L.Scott and A.P.F.Turner, Anal. Chem. 56 (1984) 667.

[2] G.Davis, Biosensors 1 (1985) 161.

[3] A.E.G.Cass, G.Davis, H.A.O.Hill and D.J.Nancarrow, Biochim. Biophys. Acta 828 (1985) 51.

[4] H.A.O.Hill and G.S.Sanghera, in A. E. G. Cass (Ed.), Biosensors, a practical approach, IRL Press, Oxford, 1990, Chapter 2,

[5] P.N.Bartlett and R.G.Whitaker, J. Electroanal. Chem. 224 (1987) 27.

[6] K.Yokoyama, E.Tamiya and I.Karube, J. Electroanal. Chem. 273 (1989) 107.

[7] W.J.Albery, P.N.Bartlett, B.J.Driscoll and R.B.Lennox, J. Electroanal. Chem. 323 (1992) 77.

[8] F.Battaglini and E.J.Calvo, Anal. Chim. Acta 258 (1992) 151.

[9] P.N.Bartlett and K.F.E.Pratt, Biosens. Bioelectron. 8 (1993) 451.

[10] S.Bacha, A.Bergel and M.Comtat, J. Electroanal. Chem. 359 (1993) 21.

[11] P.N.Bartlett and K.F.E.Pratt, J. Electroanal. Chem. 397 (1995) 61.

[12] G.Jobst, I.Moser and G.Urban, Biosens. Bioelectron. 11 (1996) 111.

[13] K.F.E.Pratt. PhD Thesis, University of Southampton, 1994.

[14] J.B.Flanagan and L.Marcoux, J. Phys. Chem. 77 (1973) 1051.

[15] K.B.Oldham, J. Electroanal. Chem. 122 (1981) 1.

[16] A.C.Michael, R.M.Wightman and C.Amatore, J. Electroanal. Chem. 267 (1989) 33.

[17] O.R.Tutty, J. Electroanal. Chem. 379 (1994) 519.

[18] J.Galceran, D.J.Gavaghan and J.S.Rollett, J. Electroanal. Chem. 394 (1995) 17.

[19] D.J.Gavaghan, J. Electroanal. Chem. 420 (1997) 147.

[20] J.Galceran, S.L.Taylor and P.N.Bartlett, J. Electroanal. Chem. 466 (1999) 15.

[21] L.Rajendran, Electrochemistry Communications 2 (2000) 679.

[22] G.Denuault. Ph.D. Thesis University of Southampton, 1989.

[23] I.Lavagnini, P.Pastore and F.Magno, J. Electroanal. Chem. 358 (1993) 193. 
[24] C.Demaille, P.R.Unwin and A.J.Bard, J. Phys. Chem. 100 (1996) 14137.

[25] L.Rajendran and M.V.Sangaranarayanan, J. Phys. Chem. B 103 (1999) 1518.

[26] J.Galceran, S.L.Taylor and P.N.Bartlett, J. Electroanal. Chem. 476 (1999) 132.

[27] O.R.Tutty and G.Denuault, IMA. J. Appl. Math. 53 (1994) 95.

[28] G.Denuault, M.Fleischmann, D.Pletcher and O.R.Tutty, J. Electroanal. Chem. 280 (1990) 243.

[29] G.L.Che and S.J.Dong, Electrochim. Acta. 37 (1992) 2695.

[30] O.R.Tutty, J. Electroanal. Chem. 377 (1994) 39.

[31] C.G.Phillips, J. Electroanal. Chem. 296 (1990) 255.

[32] K.B.Oldham, J. Electroanal. Chem. 323 (1992) 53.

[33] S.J.Dong and G.L.Che, Electrochim. Acta. 37 (1992) 2587.

[34] B.E.P.Swoboda and V.Massey, J. Biol. Chem. 240 (1965) 2209.

[35] M.Weibel and J.Bright, J. Biol. Chem. 246 (1971) 2734.

[36] A.M.Bond, K.B.Oldham and C.G.Zoski, J. Electroanal. Chem. 245 (1988) 71.

[37] Y.Saito, Rev. Polarogr. Jpn. 15 (1968) 177.

[38] G.I.Barenblatt, Dimensional Analysis, Gordon and Breach Science Publishers., New York, 1987.

[39] J.Puy, J.Galceran, J.Salvador, J.Cecilia, J.M.Diazcruz, M.Esteban and F.Mas, J. Electroanal. Chem. 374 (1994) 223.

[40] J.Galceran, J.Salvador, J.Puy, J.Cecilia, M.Esteban and F.Mas, Anal. Chim. Acta 305 (1995) 273.

[41] P.N.Bartlett and S.L.Taylor, J. Electroanal. Chem. 453 (1998) 49.

[42] D.J.Gavaghan, J. Electroanal. Chem. 456 (1998) 1.

[43] M.Fleischmann, F.Lasserre, J.Robinson and D.Swan, J. Electroanal. Chem. 177 (1984) 97.

[44] G.Denuault, Chem. Ind. London (1996) 678. 


\section{Figure Legends}

Fig. 1: Schematic representation of the homogeneous enzyme reaction at a microdisc electrode, involving three redox couples: mediator $(A / B)$, enzyme $\left(E_{R} / E_{O}\right)$ and substrate $(S / P)$.

Fig. 2: Contour profiles for species $\mathrm{S}$ (markers $\mathrm{O}$ and italic labels) and species $\mathrm{A}$ (solid lines) in a normalised coordinate system where the electrode surface corresponds to the line from $(r$ $=0, z=0)$ to $(r=1, z=0)$. Dimensionless parameters: $\gamma_{\mathrm{E}}=0.8381 ; \gamma_{\mathrm{B}}=1.4801 ; \gamma_{\mathrm{S}}=0.1194$ and $\gamma_{\mathrm{r}}=1.0205$ corresponding to $[\mathrm{B}]^{*}=4.37 \mathrm{mM} ;[\beta \text {-glucose }]^{*}=1.9 \times 10^{-3} \mathrm{M} ;\left[\mathrm{E}_{\Sigma}\right]=4.8 \mu \mathrm{M}$; $a=50 \mu \mathrm{m} ; D_{\mathrm{S}}=6.72 \times 10^{-10} \mathrm{~m}^{2} \mathrm{~s}^{-1} ; D_{\mathrm{A}}=D_{\mathrm{B}}=5.73 \times 10^{-10} \mathrm{~m}^{2} \mathrm{~s}^{-1} ; K_{\mathrm{M}}=15.9 \mathrm{mM} ; k_{\mathrm{cat}}=747$ $\mathrm{s}^{-1} ; k_{\mathrm{A}}=2.53 \times 10^{5} \mathrm{M}^{-1} \mathrm{~s}^{-1} ; m=2$ and diffusion limited conditions $\left([\mathrm{B}]^{\mathrm{e}}=0,[\mathrm{~A}]^{\mathrm{e}}=[\mathrm{B}]^{*}\right)$.

Fig. 3: Contour plot for the kinetic term $K_{\mathrm{E}}$ (given by eqn. (32)) with parameters as in Fig 2.

Fig. 4: Profile of the kinetic term $K_{\mathrm{E}}$, for the same dimensional parameters as in previous figures except $[\beta \text {-glucose }]^{*}=6.34 \times 10^{-4} \mathrm{M}$.

Fig. 5: Plot of flux against substrate concentration for three different radii (solid lines). Markers for approximation (41) with values of $S^{\mathrm{e}}$ taken from the simulation at $r=0, z=0$ ( $\square)$; at $r=1, z=0(\times)$ and their average $(\Delta)$. Other (dimensional) parameters as in Fig 2. The dashed line corresponds to approximation (42). 
Fig. 6 Plot of the approximation of equating fluxes, using eqns (44)-(45). Markers: • stands for $\bar{A}=1 / 2$ and + stands for $\bar{A}=1 / 3$. The dashed line corresponds to approximation (42). Parameters as in Fig 2 except $a=12.5 \mu \mathrm{m}$.

Fig. 7: Flux in terms of the logarithm of the concentration of mediator. The asymptotic pseudo-first order EC' value for low mediator concentration is depicted by the horizontal dashed line. The vertical dotted line indicates the limit for EC' as estimated by eqn. (49). The approximately parabolic discontinuous line on the right corresponds to the approximation (42). Parameters as in Fig 2 except $a=12.5 \mu \mu$ and $[\beta \text {-glucose }]^{*}=41.5 \mathrm{mM}$.

Fig. 8: Effect of the mediator concentration, the substrate concentration and electrode radius on the normalised current: a) $[\mathrm{B}]^{*}=4.37 \mathrm{mM}$, b) $[\mathrm{B}]^{*}=0.437 \mathrm{mM}$ and c) $[\mathrm{B}]^{*}=0.0437 \mathrm{mM}$, for $a=25 \mu \mathrm{m}$ (marker $\Delta$ ), $a=12.5 \mu \mathrm{m}(\square)$, and $a=5 \mu \mathrm{m}(\mathrm{O})$ microdisc electrodes. Other parameters as in Fig 2. The dashed horizontal lines with the same small markers show the pseudo-first order EC' limit computed exactly [26].

Fig. 9: Effect of the radius $a$ on the dimensionless current $(\phi)$ for a case with a small amount of mediator $\left([\mathrm{B}]^{*}=0.0437 \mathrm{mM}\right)$ and large amount of substrate $\left([\beta \text {-glucose }]^{*}=41.5 \mathrm{mM}\right)$. Other parameters as in Fig 2. Markers $O$ correspond to the FEM simulation. The solid straight line corresponds to the application of eqn. (53). 
Fig. 10: Effect of changing the kinetic parameters on the plot of flux against substrate concentration. In each graph, one constant is increased 20\% (marker O) or decreased 20\% (marker $\times)$ with respect to the reference value used in all previous figures: a) Effect of $k_{\mathrm{A}}$ (maker $\bigcirc$ for $k_{\mathrm{A}}=3.04 \times 10^{5} \mathrm{M}^{-1} \mathrm{~s}^{-1}$; marker $\times$ for $k_{\mathrm{A}}=2.02 \times 10^{5} \mathrm{M}^{-1} \mathrm{~s}^{-1}$ ), b) Effect of $k_{\text {cat }}$ (marker $\bigcirc$ for $k_{\text {cat }}=896 \mathrm{~s}^{-1} ;$ marker $\times$ for $\left.k_{\text {cat }}=598 \mathrm{~s}^{-1}\right)$, and c) $K_{\mathrm{M}}\left(\right.$ maker $\bigcirc$ for $K_{\mathrm{M}}=19.1$ $\mathrm{mM}$; marker $\times$ for $\left.K_{\mathrm{M}}=12.7 \mathrm{mM}\right)$. Other parameters as in Fig 2 except $a=12.5 \mu \mathrm{m}$.

Fig. 11: Comparison of experimental data with simulated data for a mediator concentration of 4.37 mM. Radii: $a=12.5 \mu \mathrm{m}$ (marker o) and $a=5 \mu \mathrm{m}(\square)$. Other parameters as in Fig 2 [11].

Fig. 12: Comparison of experimental data with simulated data for a mediator concentration of $1 \mathrm{mM}$. Radii: $a=25 \mu \mathrm{m}$ (marker $\Delta$ ), $a=12.5 \mu \mathrm{m}(\square)$, and $a=5 \mu \mathrm{m}(\mathbf{0})$. Other parameters as in Fig 2. 


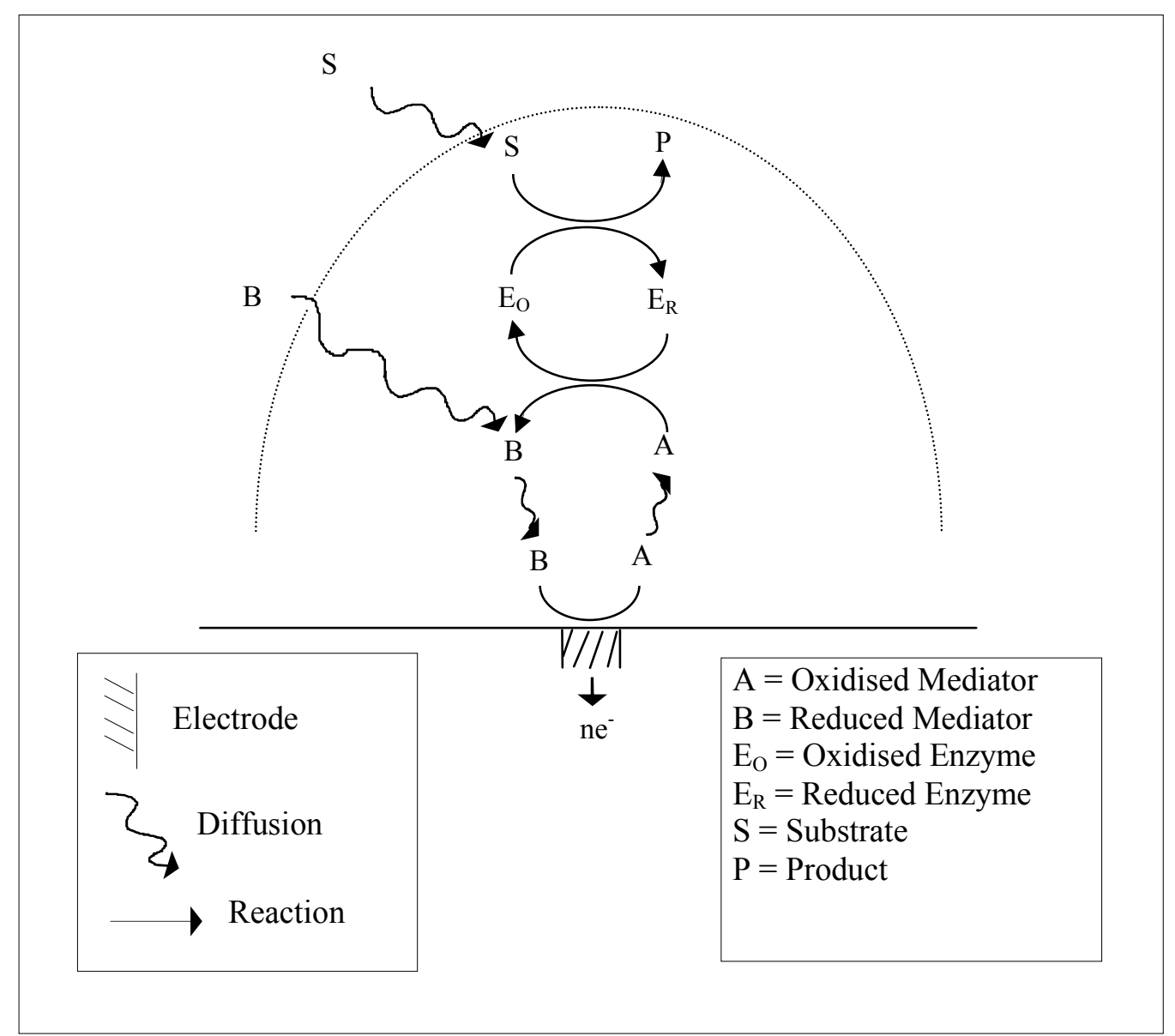

Fig 1 


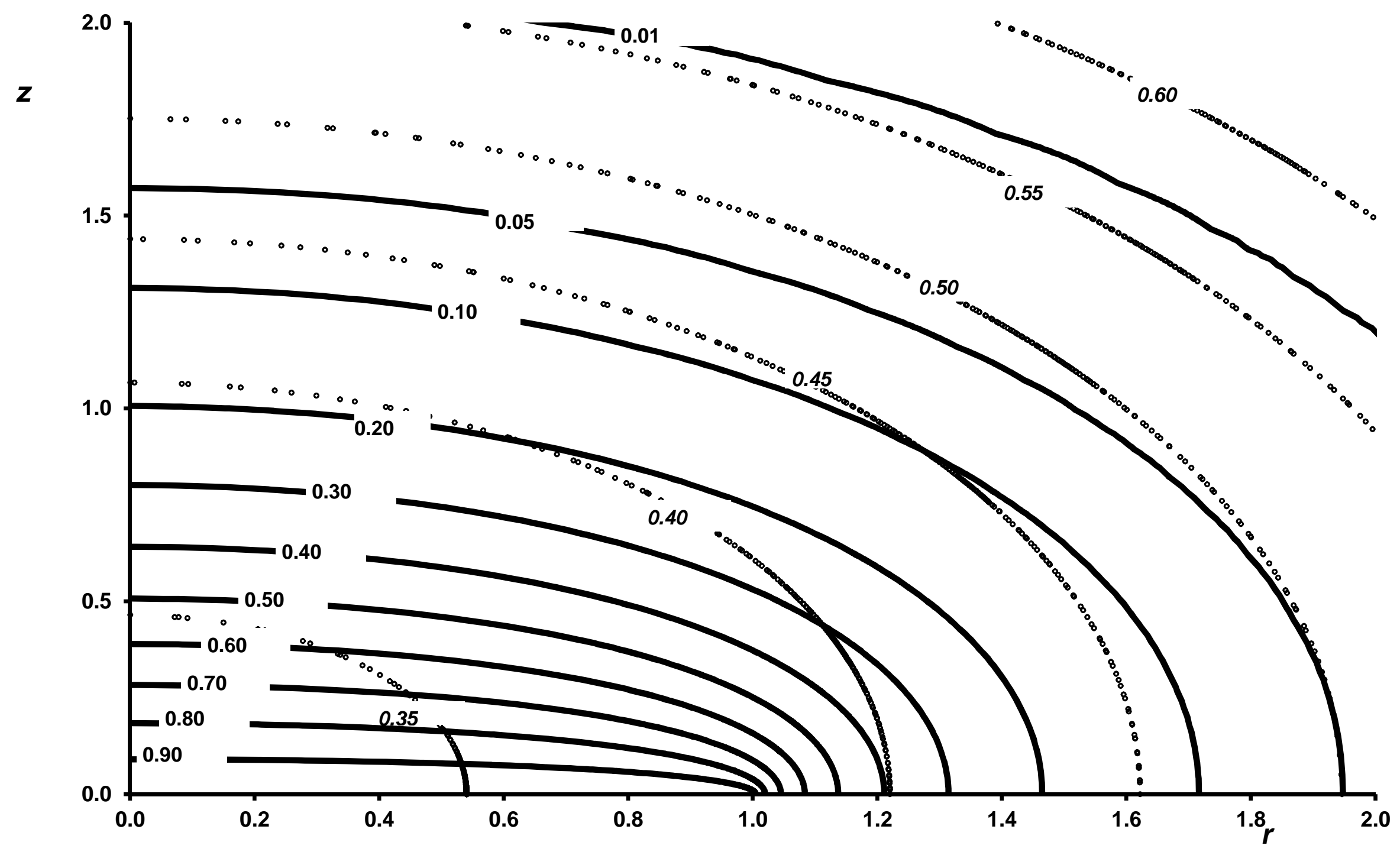

Fig 2 


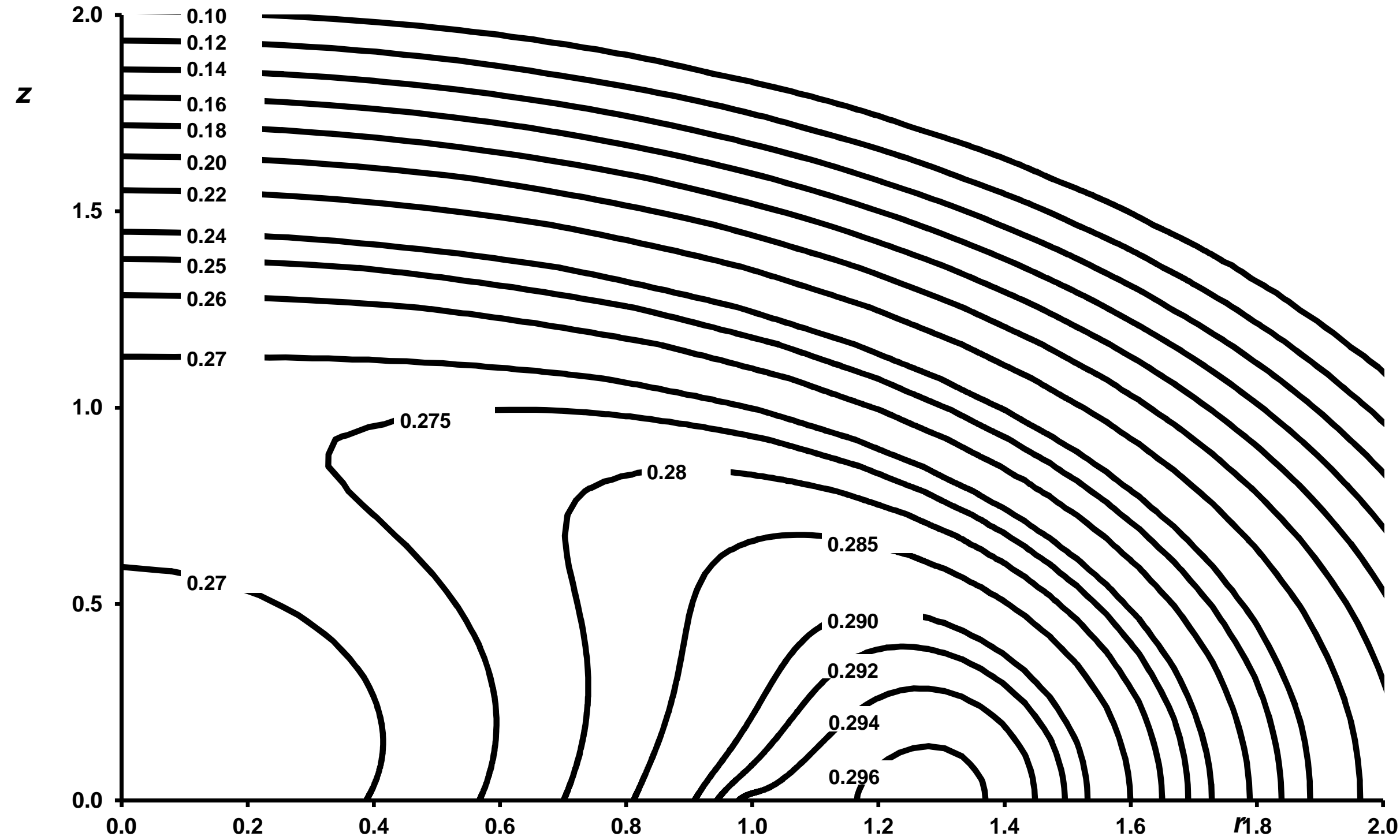




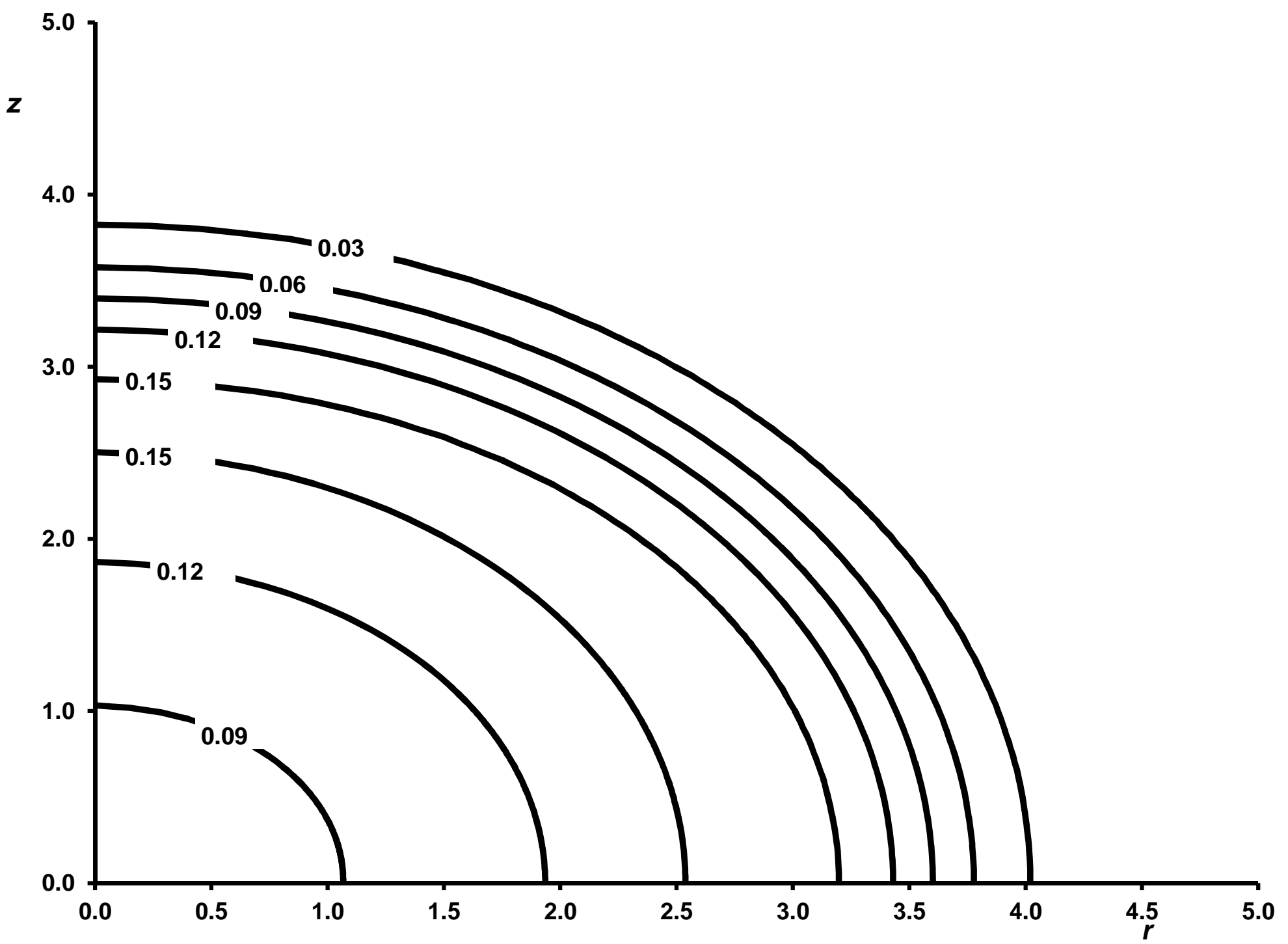

Fig 4 


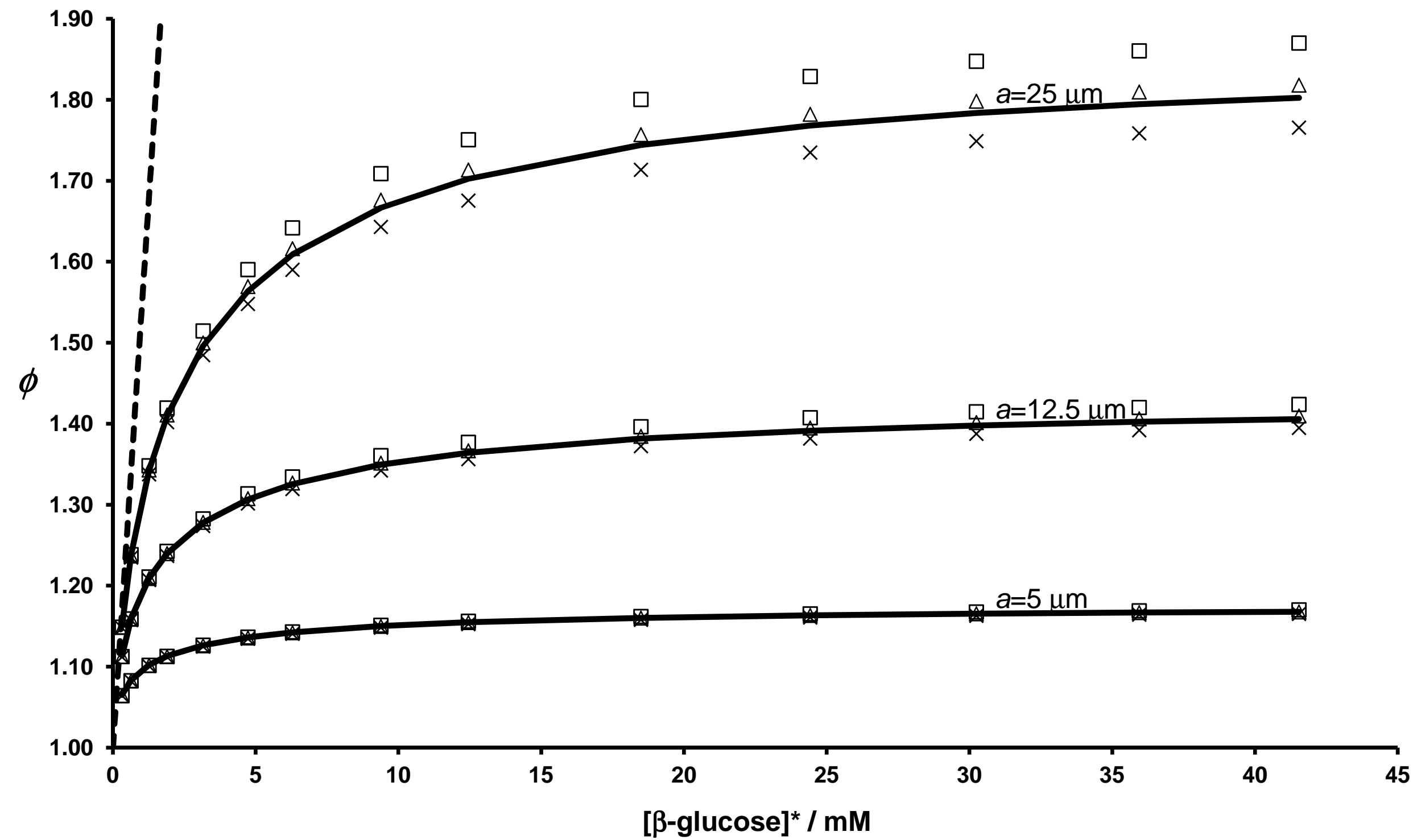

Fig 5 


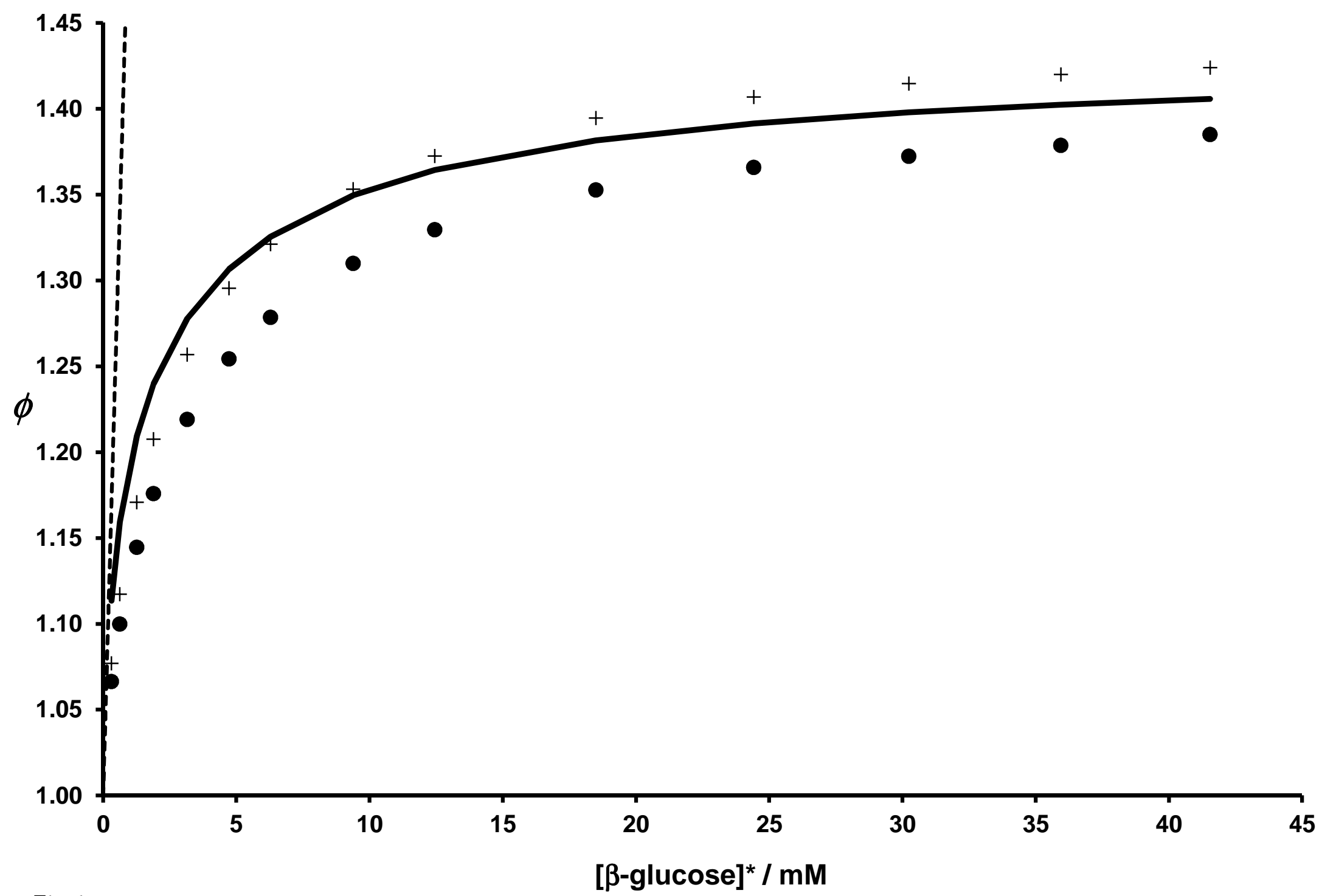

Fig 6 


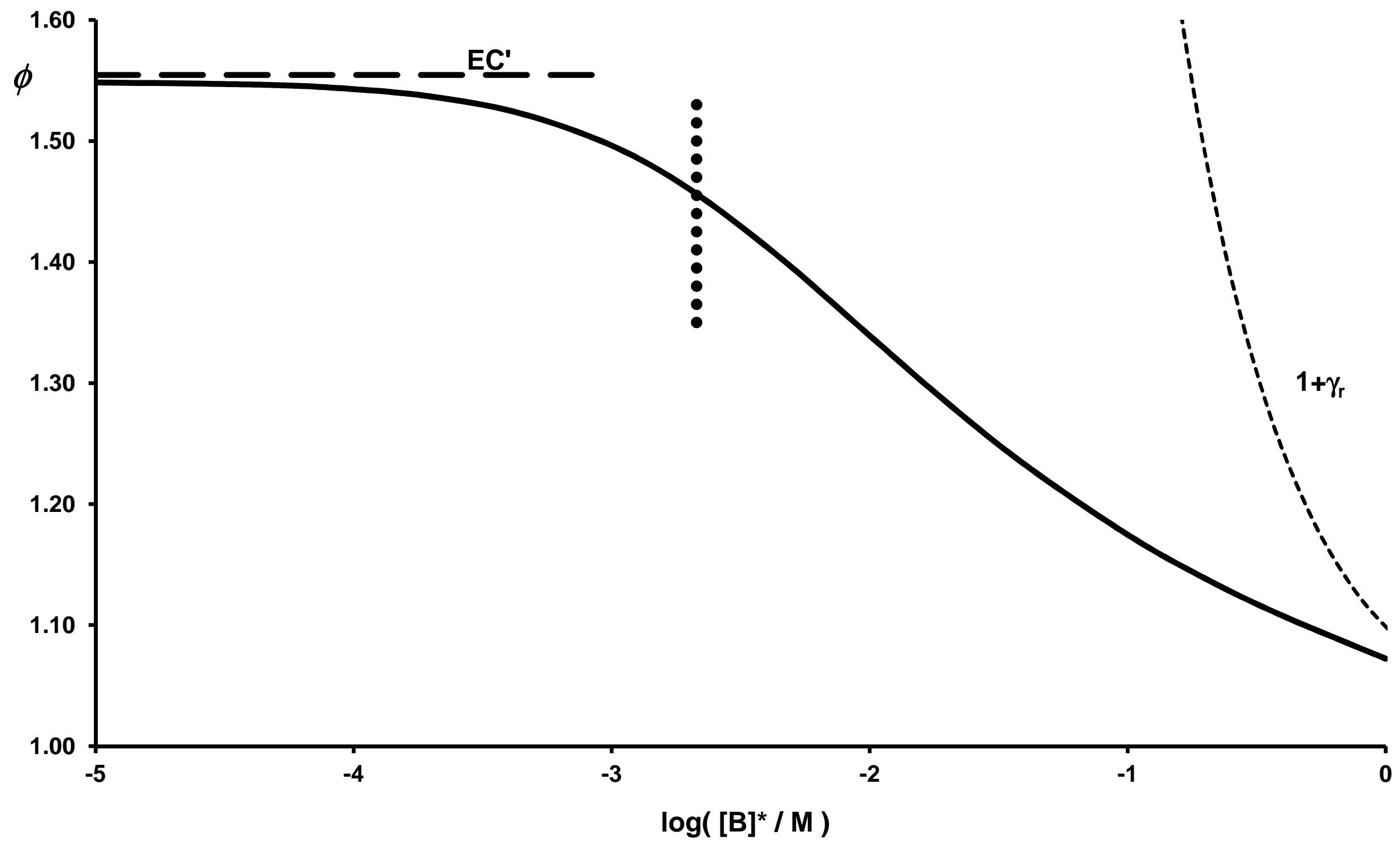

Fig 7 

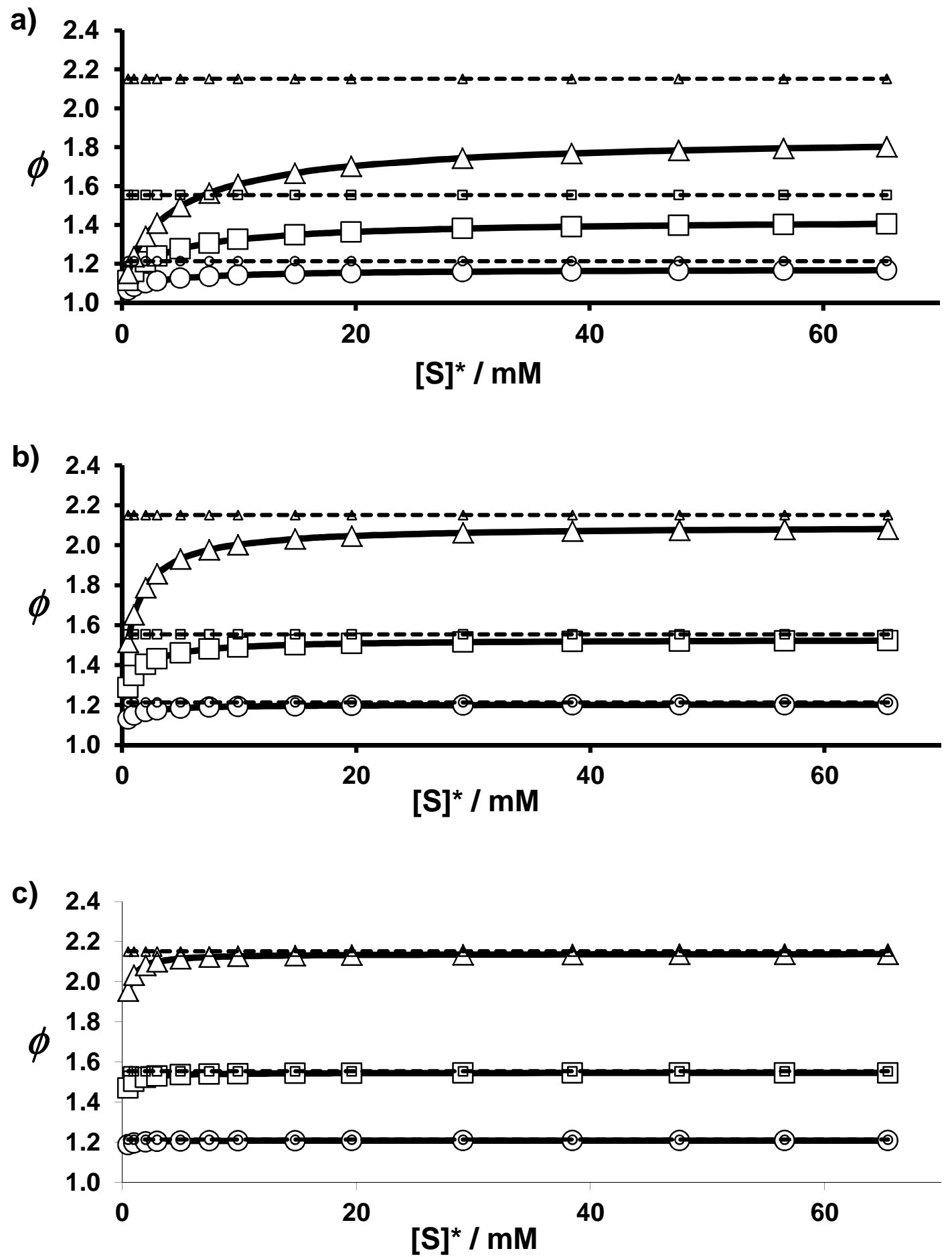

Fig 8 


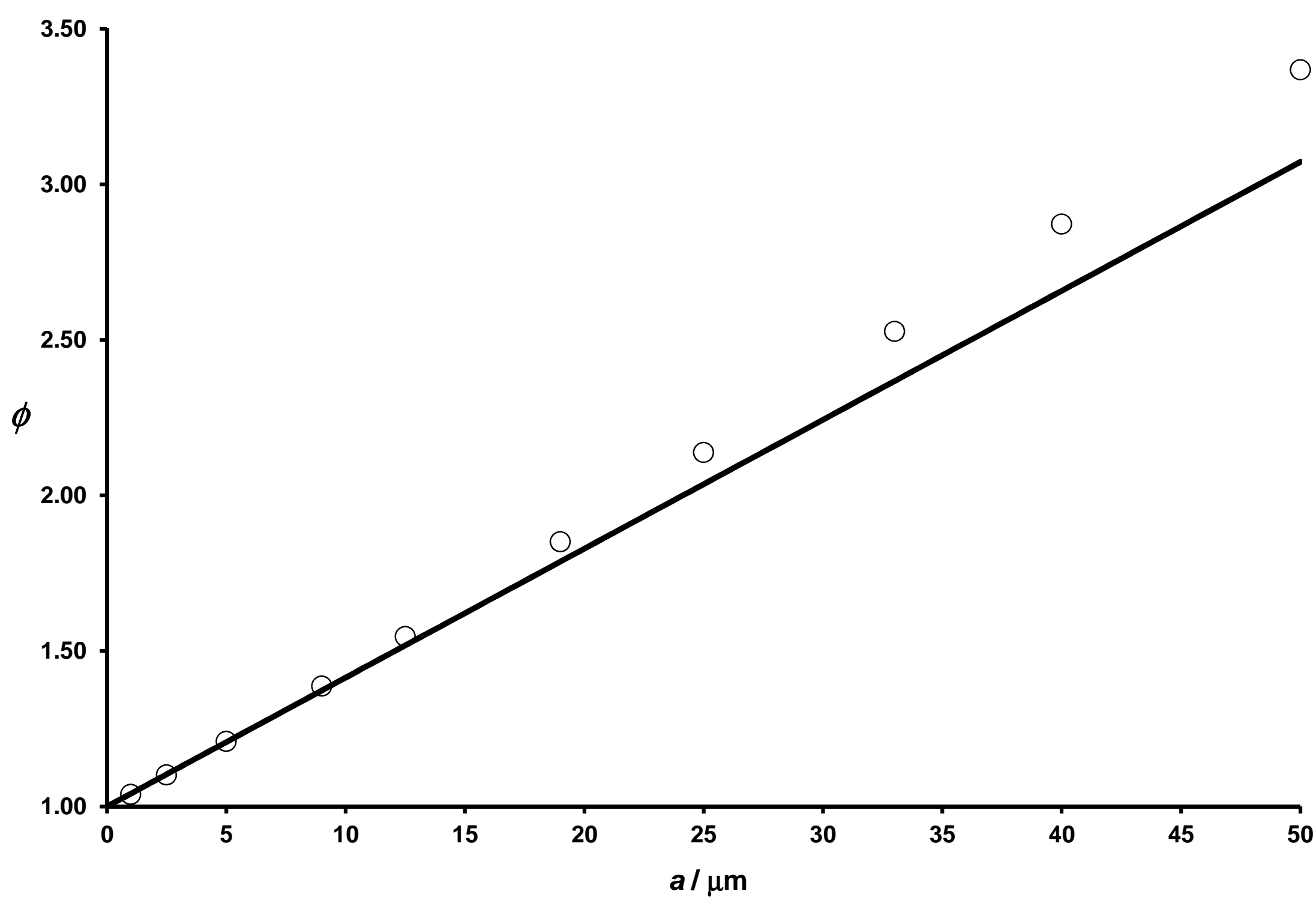

Fig 9 

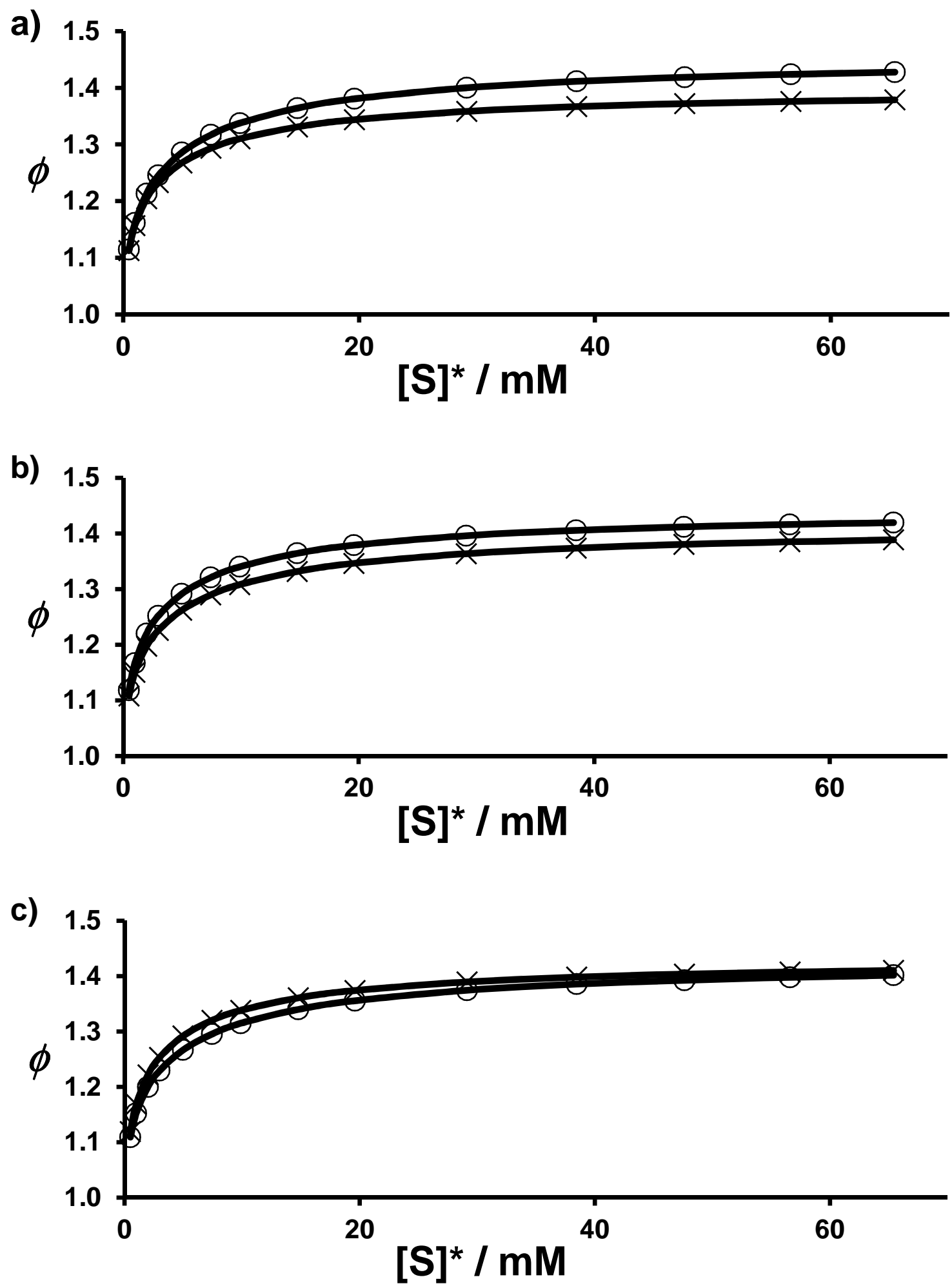

Fig 10 


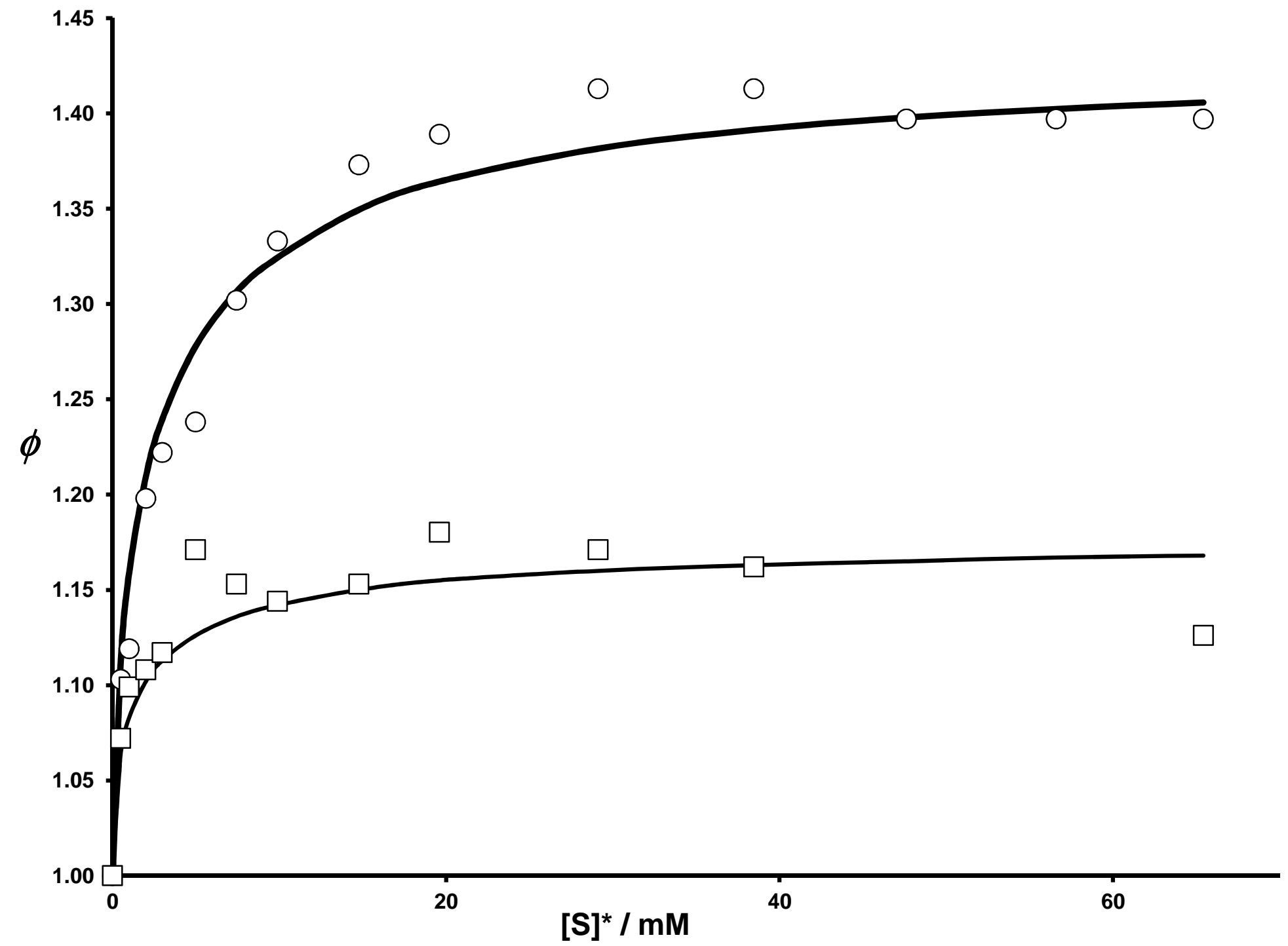

Fig 11 


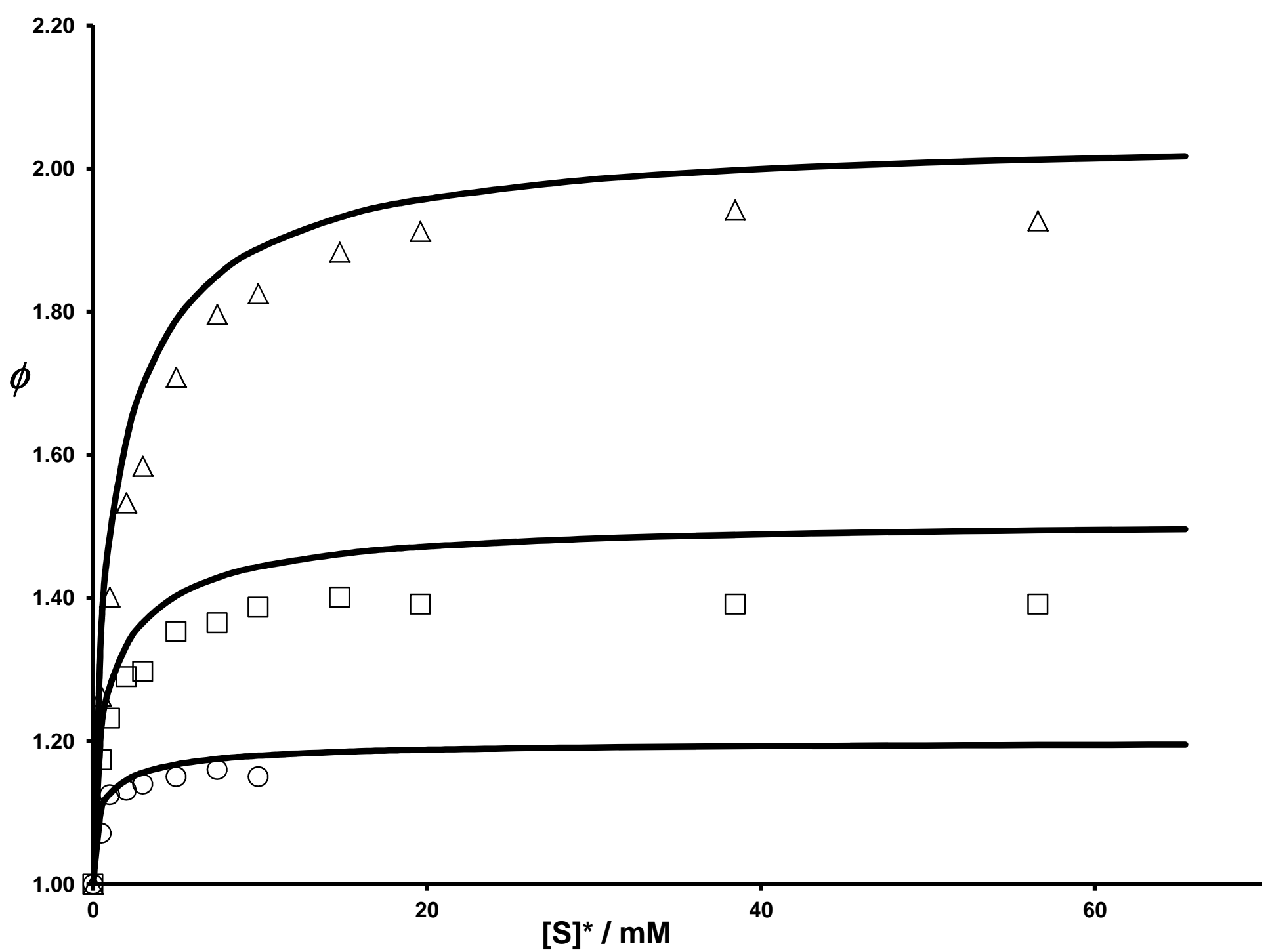

Fig 12 